Article

\title{
Acting Semicircular Elements Induced by Orthogonal Projections on Von-Neumann-Algebras
}

\author{
Ilwoo Cho \\ Department of Mathematics, Saint Ambrose University, 421 Ambrose Hall, 518 W. Locust St., \\ Davenport, IA 52803, USA; choilwoo@sau.edu
}

Received: 18 September 2017; Accepted: 21 November 2017; Published: 6 December 2017

\begin{abstract}
In this paper, we construct a free semicircular family induced by $|\mathbb{Z}|$-many mutually-orthogonal projections, and construct Banach *-probability spaces containing the family, called the free filterizations. By acting a free filterization on fixed von Neumann algebras, we construct the corresponding Banach $*$-probability spaces, called affiliated free filterizations. We study free-probabilistic properties on such new structures, determined by both semicircularity and free-distributional data on von Neumann algebras. In particular, we study how the freeness on free filterizations, and embedded freeness conditions on fixed von Neumann algebras affect free-distributional data on affiliated free filterizations.
\end{abstract}

Keywords: free probability; Banach $*$-probability spaces; von Neumann algebras; projections; semicircular elements; free filterizations; affiliated free filterizations

JEL Classification: 11R56; 46L10; 46L40; 46L53; 46L54; 47L15; 47L30; 47L55

\section{Introduction}

There are different approaches to construct semicircular elements (e.g., [1-3]) in topological *-probability spaces (e.g., $C^{*}$-probability spaces, or $W^{*}$-probability spaces, or Banach $*$-probability spaces, etc.). In [4], we introduced how to construct semicircular elements in certain topological *-probability spaces. The construction of [4] is highly motivated by that of weighted-semicircular elements in a Banach $*$-probability space in the sense of $[5,6]$. In this paper, we put our semicircular elements on a fixed $W^{*}$-probability space, and then consider structure theorems of such Banach *-probabilistic structures under our actions, and study free-distributional data from the structures.

\subsection{Motivation and Background}

The main purpose of this paper are (i) to construct (weighted-)semicircular elements from orthogonal projections, (ii) to act them to von Neumann algebras, and (iii) to study free-distributional data determined both by these (weighted-)semicircular elements, and free distributions on von Neumann algebras. In particular, the construction of our (weighted-)semicircular elements are highly motivated by the constructions of $[5,6]$.

In [7], the author and Gillespie studied free-probabilistic models of certain embedded sub-structures of Hecke algebras $\mathcal{H}\left(G_{p}\right)$ generated by the generalized linear groups $G_{p}=G L_{2}\left(\mathbb{Q}_{p}\right)$ over $p$-adic number fields $\mathbb{Q}_{p}$, for fixed primes $p$. In addition, such a free-probabilistic model is generalized in [8] fully on $\mathcal{H}\left(G_{p}\right)$. Motivated by $[7,8]$, independently, the author mimicked the techniques and ideas to construct weighted-semicircular elements and corresponding semicircular elements induced by certain orthogonal projections on $\mathbb{Q}_{p}$ in [6]. In [5], as an application of the main results of [6], we studied free stochastic calculus for the weighted-semicircular laws in the sense of [6].

Our constructions of weighted-semicircular, and semicircular elements in this paper is understood as a pure operator-theoretic version of those of $[5,6]$. 


\subsection{Overview}

Here, we generalize the free probability on free filterizations (which are Banach $*$-probability spaces generated by the semicircular elements obtained in [4]). By using these free filterizations to arbitrarily fixed von Neumann algebras $M$, we consider $M$-affiliated free filterizations, and establish suitable free-probabilistic models on them.

In Section 2, we briefly mention background theories for our proceeding works.

In Section 3, we introduce fundamental free-probabilistic settings from given $|\mathbb{Z}|$-many mutually orthogonal projections.

In Sections 4 and 5, we construct weighted-semicircular, and semicircular elements induced by given orthogonal projections.

In Section 5, from the ingredients of Sections 3, 4 and 5, we construct free filterizations as free product Banach $*$-probability spaces, and consider fundamental free-distributional data on them.

In Section 7, we act an arbitrarily fixed free filterization to a given von Neumann algebra, and construct the corresponding von-Neumann-algebra-affiliated free filterizations, and study how our semicircular elements work on such structures.

In Section 8, from the free-distributional data obtained in Section 7, we construct-and-study weighted-semicircular, and semicircular elements in affiliated free filterizations. By doing that, one can see how the freeness on our free filterizations affects the free probability on affiliated structures.

In Section 9, by considering (embedded, or full) freeness conditions on given von Neumann algebras, free-distributional data on affiliated free filterizations are studied. We show how the freeness conditions on von Neumann algebras affect the affiliated structures.

In Section 10, an example for the main results of Sections 7, 8 and 9 will be considered. In particular, we are interested in the case where a fixed von Neumann algebra is given to be a free group factor $L\left(F_{n}\right)$ (e.g., [1]) generated by the free group $F_{n}$ with $n$-generators.

\section{Preliminaries}

Readers can check fundamental analytic-and-combinatorial free probability theory from [2,3,9] (and cited papers therein). Free probability is understood as the noncommutative operator-algebraic version of classical probability theory and statistics. The classical independence is replaced by the freeness, by replacing measures on sets to linear functionals on (noncommutative algebraic, or topological $*-$ ) algebras. It has various applications not only in pure mathematics (e.g., [1,2]), but also in related topics (e.g., [8] through [7]). In particular, we will use combinatorial free-probabilistic approach of Speicher (e.g., [9]). Free moments andfree cumulantsof operators (representing free-distributional data of operators), or free probability spaces, or free product of algebras will be considered without introducing detailed concepts.

\section{Certain Banach $*$-Algebras Induced by Orthogonal Projections}

Let $(A, \psi)$ be a topological $*$-probability space $\left(C^{*}\right.$-probability space, or $W^{*}$-probability space, or Banach $*$-probability space, etc.) of a topological $*$-algebra $A\left(C^{*}\right.$-algebra, resp., von Neumann algebra, resp., Banach $*$-algebra), and a bounded linear functional $\psi$ on $A$.

An operator $a$ of $A$ is said to be a free random variable whenever it is regarded as an element of $(A, \psi)$. As usual, we say $a$ is self-adjoint (as an operator in $A$ ), if $a^{*}=a$ in $A$, where $a^{*}$ is the adjoint of $a$.

Definition 1. A self-adjoint free random variable a is said to be weighted-semicircular in $(A, \psi)$ with its weight $t_{0} \in \mathbb{C}^{\times}=\mathbb{C} \backslash\{0\}$, (or, in short, $t_{0}$-semicircular), if a satisfies the free-cumulant computation,

$$
k_{n}^{\psi}(a, \ldots, a)= \begin{cases}k_{2}^{\psi}(a, a)=t_{0} & \text { if } n=2, \\ 0 & \text { otherwise }\end{cases}
$$

for all $n \in \mathbb{N}$, where $k_{n}^{\psi}(\ldots)$ is the free cumulant on $A$ in terms of $\psi$, under the Möbius inversion of [9].

If $t_{0}=1$ in (3.1), the 1-semicircular element a is simply said to be semicircular in $(A, \psi)$, 
By definition, a free random variable $a$ is semicircular in $(A, \psi)$, if $a$ satisfies

$$
k_{n}^{\psi}(a, \ldots, a)= \begin{cases}1 & \text { if } n=2, \\ 0 & \text { otherwise, }\end{cases}
$$

for all $n \in \mathbb{N}$.

By the Möbius inversion of [9], one can characterize the weighted-semicircularity (3.1) as follows: $a$ is $t_{0}$-semicircular in $(A, \psi)$, if and only if

$$
\psi\left(a^{n}\right)=\omega_{n}\left(t_{0}^{\frac{n}{2}} c_{\frac{n}{2}}\right),
$$

where

$$
\omega_{n} \stackrel{\text { def }}{=} \begin{cases}1 & \text { if } n \text { is even, } \\ 0 & \text { if } n \text { is odd, }\end{cases}
$$

for all $n \in \mathbb{N}$, where $c_{k}$ are the $k$-th Catalan numbers,

$$
c_{k}=\frac{1}{k+1}\left(\begin{array}{c}
2 k \\
k
\end{array}\right)=\frac{1}{k+1} \frac{(2 k) !}{k ! k !}=\frac{(2 k) !}{k !(k+1) !},
$$

for all $k \in \mathbb{N}_{0}=\mathbb{N} \cup\{0\}$.

By (3.2) and (3.3), a free random variable $a$ is semicircular in $(A, \psi)$, if and only if

$$
\psi\left(a^{n}\right)=\omega_{n} c_{\frac{n}{2}},
$$

for all $n \in \mathbb{N}$, where $\omega_{n}$ are in the sense of (3.3).

Thus, one can use the $t_{0}$-semicircularity (3.1) (respectively, the semicircularity (3.2)), and its characterization (3.3) (respectively, (3.4)) alternatively.

Recall that, if a free random variable $x \in(A, \psi)$ is self-adjoint, then the sequences

$$
\left(\psi\left(x^{n}\right)\right)_{n=1}^{\infty} \text {, and }\left(k_{n}^{\psi}(x, \ldots, x)\right)_{n=1}^{\infty}
$$

provide equivalent free distributions of $x$.

Indeed, the Möbius inversion (of [9]) satisfies

$$
\psi\left(a^{n}\right)=\sum_{\pi \in N C(n)}\left(\prod_{V \in \pi} k_{|V|}(a, \ldots, a)\right),
$$

and

$$
k_{n}^{\psi}(a, \ldots, a)=\sum_{\pi \in N C(n)}\left(\prod_{V \in \theta} \psi\left(a^{|V|}\right)\right) \mu\left(\pi, 1_{n}\right),
$$

where $N C(n)$ is the lattice of all noncrossing partitions over $\{1, \ldots, n\}$, and " $V \in \pi^{\prime \prime}$ means " $V$ is a block of $\pi, "$ and where $\mu$ is the Möbius functional in the incidence algebra over

$$
\cup_{n \in \mathbb{N}}(N C(n) \times N C(n))
$$

(see [9]).

Now, let $A$ be a given $C^{*}$-algebra, and let $q_{j} \in A$ be a projection in the sense that:

$$
q_{j}^{*}=q_{j}=q_{j}^{2} \text { in } A,
$$

for all $j \in \mathbb{Z}$, where $\mathbb{Z}$ is the set of all integers. Moreover, assume that the projections $\left\{q_{j}\right\}_{j \in \mathbb{Z}}$ are mutually orthogonal from each other in the sense that: 


$$
q_{i} q_{j}=\delta_{i, j} q_{j} \text { in } A, \text { for all } i, j \in \mathbb{Z},
$$

where $\delta$ means the Kronecker delta.

Remark 1. Such mutually orthogonal $|\mathbb{Z}|$-many projections $\left\{q_{j}\right\}_{j \in \mathbb{Z}}$ can be found in a $C^{*}$-algebra $A$, naturally, or artificially. One can find such projections naturally as in $[3,4]$.

If $\left\{q_{j}\right\}_{j=1}^{N}$ is a finite family of mutually orthogonal projections in a certain $C^{*}$-algebra $A_{0}$, for some $N \in \mathbb{N}$, then one can construct a $C^{*}$-algebra $A$,

$$
A=A_{0}^{\oplus|\mathbb{Z}|}=\cdots \oplus A_{0} \oplus A_{0} \oplus A_{0} \oplus \cdots,
$$

under product topology, and then we obtain the mutually orthogonal $|\mathbb{Z}|$-many projections,

$$
\left\{\cdots,\left\{q_{j}\right\}_{j=1}^{N},\left\{q_{j}\right\}_{j=1}^{N},\left\{q_{j}\right\}_{j=1}^{N}, \cdots\right\}
$$

in $A$, artificially.

Similarly, if $N=\infty$, and $\left\{q_{j}\right\}_{j=1}^{\infty}$ forms a family of mutually orthogonal projections in a certain $C^{*}$-algebra $A_{0}$, then one can construct a $C^{*}$-algebra $A$,

$$
A=A_{0} \oplus A_{0}
$$

with a family of mutually orthogonal $|\mathbb{Z}|$-many projections,

$$
\left\{\left\{\cdots, q_{3}, q_{2}, q_{1}\right\},\left\{q_{1}, q_{2}, q_{2}, \cdots\right\}\right\},
$$

in $A$, artificially.

Therefore, from below, we always assume a given $C^{*}$-algebra $A$ has a family $\left\{q_{j}\right\}_{j \in \mathbb{Z}}$ of mutually orthogonal $|\mathbb{Z}|$-many projections.

Note that we are not interested in operator-algebraic structures or properties of $A$, but interested in induced weighted-semicircularity or semicircularity from projections in a $C^{*}$-algebra $A$.

Now, we fix a family $\left\{q_{j}\right\}_{j \in \mathbb{Z}}$ of mutually orthogonal projections of a fixed $C^{*}$-algebra $A$, and we denote it by $\mathbf{Q}$;

satisfying (3.5).

$$
\mathbf{Q}=\left\{q_{j}: j \in \mathbb{Z}\right\} \text { in } A,
$$

In addition, let $Q$ be the $C^{*}$-subalgebra of $A$ generated by $\mathbf{Q}$ of (3.6),

$$
Q \stackrel{\text { def }}{=} C^{*}(\mathbf{Q}) \subseteq A
$$

Then, it is easy to get the following structure theorem.

Proposition 1. Let $Q$ be a $C^{*}$-subalgebra (3.7) of a given $C^{*}$-algebra $A$, generated by the family $\mathbf{Q}$ of (3.6). Then,

$$
Q \stackrel{*-i s o}{=} \bigoplus_{j \in \mathbb{Z}}\left(\mathbb{C} \cdot q_{j}\right) \stackrel{*-i s o}{=} \mathbb{C} \oplus|\mathbb{Z}|, \text { in } A
$$

Proof. The structure theorem (3.8) is proven by the mutual-orthogonality (3.5) of the generator set $\mathbf{Q}$ of (3.6) in $A$.

Now, assume that we fix a bounded linear functional $\psi$ on the $C^{*}$-algebra $A$, creating the corresponding $C^{*}$-probability space $(A, \psi)$. From this fixed $C^{*}$-probability space $(A, \psi)$, define now linear functionals $\psi_{j}$ on $Q$ by

$$
\psi_{j}\left(q_{i}\right)=\delta_{i j} \psi\left(q_{j}\right), \text { for all } i \in \mathbb{Z},
$$


for all $j \in \mathbb{Z}$, where $\psi$, on the right-hand side of (3.9), is the restricted linear functional of $\psi$ on the $C^{*}$-subalgebra $Q$ of $A$. Remark that such linear functionals $\left\{\psi_{j}\right\}_{j \in \mathbb{Z}}$ of (3.9) are well-defined on $Q$ by (3.8).

Therefore, if $q \in Q$, then

$$
\left.q=\sum_{j \in \mathbb{Z}} t_{j} q_{j} \quad \text { (with } t_{j} \in \mathbb{C}\right)
$$

and, hence,

$$
\psi_{j}(q)=\psi_{j}\left(t_{j} q_{j}\right)=t_{j} \psi\left(q_{j}\right),
$$

by the definition (3.9) of $\psi_{j}$, for all $j \in \mathbb{Z}$. It shows that the system $\left\{\psi_{j}\right\}_{j \in \mathbb{Z}}$ of the linear functionals (3.9) filterize, or sectionize $Q$ free-probabilistically.

Definition 2. The $C^{*}$-probability spaces $\left(Q, \psi_{j}\right)$ are called the $j$-th $C^{*}$-probability spaces of $Q$ in $(A, \psi)$, where $Q$ is the $C^{*}$-subalgebra (3.7) of $A$, and $\psi_{j}$ are in the sense of (3.9), for all $j \in \mathbb{Z}$.

Now, let us define bounded linear transformations $c$ and $a$ "acting on the $C^{*}$-algebra $Q^{\text {" by }}$

$$
c\left(q_{j}\right)=q_{j+1}, \text { and } a\left(q_{j}\right)=q_{j-1},
$$

for all $j \in \mathbb{Z}$. Then, $c$ and $a$ are indeed well-defined bounded linear operators "on $Q$," understood as elements of the operator space $B(Q)$, consisting of all bounded linear transformations on $Q$ (e.g., [10]). Without loss of generality, one can regard $c$ and $a$ of (3.10) as Banach-space operators on a Banach space $Q$.

Definition 3. We call these Banach-space operators $c$ and a of (3.10), the creation, respectively, the annihilation on $Q$.

Define now a new Banach-space operator $l$ in the operator space $B(Q)$ by

$$
l=c+a \text { on } Q,
$$

where $c$ and $a$ are the creation, respectively, the annihilation on $Q$.

Definition 4. We call the Banach-space operator lof (3.11), the radial operator on $Q$.

By the definition (3.11), one has

$$
l\left(\sum_{j \in \mathbb{Z}} t_{j} q_{j}\right)=\sum_{j \in \mathbb{Z}} t_{j}\left(q_{j+1}+q_{j-1}\right) .
$$

Now, define a Banach subspace

$$
\mathfrak{L} \stackrel{\text { def }}{=} \overline{\mathbb{C}[\{l\}]}]^{\|\cdot\|},
$$

of $B(Q)$, generated by the radial operator $l$, equipped with the operator norm,

$$
\|T\|=\sup \left\{\|T q\|_{Q}:\|q\|_{Q}=1\right\},
$$

on $B(Q)$, where $\|\cdot\|_{Q}$ is the $C^{*}$-norm on $Q$, where $\bar{X}^{\|\cdot\|}$ mean the operator-norm closures of subsets $X$ in $B(Q)$. By the definition (3.12), it is not difficult to see that this Banach-subspace $\mathfrak{L}$ forms a Banach algebra inside $B(Q)$.

On the Banach algebra $\mathfrak{L}$ of (3.12), define a unary operation $(*)$ by

$$
\left(\sum_{n=0}^{\infty} t_{n} l^{n}\right)^{*}=\sum_{n=0}^{\infty} \overline{t_{n}} l^{n} \text { in } \mathfrak{L},
$$


where $\bar{z}$ are the conjugates of $z$ in $\mathbb{C}$.

Then, the operation (3.13) is a well-defined adjoint on $\mathfrak{L}$, and hence, all elements of $\mathfrak{L}$ are adjointable (in the sense of [10]) in $B(Q)$. Thus, the Banach algebra $\mathfrak{L}$ of (3.12) forms a Banach $*$-algebra.

Definition 5. We call the Banach $*$-algebra $\mathfrak{L}$ of (3.12), the radial (Banach $*$-)algebra on $Q$.

Now, let $\mathfrak{L}$ be the radial algebra on $Q$. Construct now the tensor product Banach $*$-algebra,

$$
\mathfrak{L}_{Q}=\mathfrak{L} \otimes_{\mathbb{C}} Q .
$$

Definition 6. We call the tensor product Banach $*$-algebra $\mathfrak{L}_{Q}$ of (3.14), the radial projection (Banach $*$-)algebra on $Q$.

\section{Weighted-Semicircular Elements Induced by $Q$}

Throughout this section, we fix the settings of Section 3, and construct weighted-semicircular elements induced by the family $\mathbf{Q}$ of mutually orthogonal $|\mathbb{Z}|$-many projections in a fixed $C^{*}$-probability space $(A, \psi)$. Let $\left(Q, \psi_{j}\right)$ be $j$-th $C^{*}$-probability space of $Q$ in $(A, \psi)$, where $\psi_{j}$ are the linear functionals (3.9), for all $j \in \mathbb{Z}$, and let $\mathfrak{L}_{Q}$ be the radial projection algebra (3.14) on $Q$.

Remark that, if

then

$$
u_{j}=l \otimes q_{j} \in \mathfrak{L}_{Q}, \text { for all } j \in \mathbb{Z},
$$

$$
u_{j}^{n}=\left(l \otimes q_{j}\right)^{n}=l^{n} \otimes q_{j}, \text { for all } n \in \mathbb{N},
$$

since $q_{j}^{n}=q_{j}$, for all $n \in \mathbb{N}$, for $j \in \mathbb{Z}$.

Then, one can construct a linear functional $\varphi_{j}$ on the radial projection algebra $\mathfrak{L}_{Q}$ by a linear morphism satisfying that

$$
\varphi_{j}\left(\left(l \otimes q_{i}\right)^{n}\right)=\varphi_{j}\left(l^{n} \otimes q_{i}\right) \stackrel{\text { def }}{=} \psi_{j}\left(l^{n}\left(q_{i}\right)\right),
$$

for all $n \in \mathbb{N}$, for all $i, j \in \mathbb{Z}$. Note that such linear functionals $\varphi_{j}$ of (4.2) are well-defined by (3.8) and (3.14).

Definition 7. We call the Banach $*$-probability spaces,

$$
\left(\mathfrak{L}_{Q}, \varphi_{j}\right), \text { for all } j \in \mathbb{Z},
$$

the $j$-th (Banach-*-)probability spaces on $Q$.

Now, consider the elements $l^{n}\left(q_{i}\right)$ in $Q$, for all $n \in \mathbb{N}, i \in \mathbb{Z}$. Observe first that, if $c$ and $a$ are the creation, respectively, the annihilation on $Q$ in the sense of (3.10), then

$$
c a=1_{Q}=a c,
$$

where $1_{Q}$ is the identity operator on $Q$ in the operator space $B(Q)$, satisfying

$$
1_{Q}(q)=q, \text { for all } q \in Q .
$$

Indeed, for any $q_{j} \in \mathbf{Q}$ in $Q$,

$$
c a\left(q_{j}\right)=c\left(a\left(q_{j}\right)\right)=c\left(q_{j-1}\right)=q_{j-1+1}=q_{j},
$$

and

$$
a c\left(q_{j}\right)=a\left(c\left(q_{j}\right)\right)=a\left(q_{j+1}\right)=q_{j+1-1}=q_{j},
$$


for all $j \in \mathbb{Z}$.

By (4.4), one can get that

$$
c^{n} a^{n}=1_{Q}=a^{n} c^{n}, \text { for all } n \in \mathbb{N}
$$

and

$$
c^{n_{1}} a^{n_{2}}=a^{n_{2}} c^{n_{1}}, \text { for all } n_{1}, n_{2} \in \mathbb{N} .
$$

Furthermore, since the radial algebra $\mathfrak{L}$, which is a tensor-factor of $\mathfrak{L}_{Q}$, is generated by a single generator $l$, one has

in $\mathfrak{L}$, for all $n \in \mathbb{N}$, by (4.4) and $(4.4)^{\prime}$, where

$$
l^{n}=(c+a)^{n}=\sum_{k=0}^{n}\left(\begin{array}{c}
n \\
k
\end{array}\right) c^{k} a^{n-k}
$$

$$
\left(\begin{array}{l}
n \\
k
\end{array}\right)=\frac{n !}{k !(n-k !)}, \text { for all } k \leq n \in \mathbb{N}_{0} .
$$

Note that, for any $n \in \mathbb{N}$,

$$
l^{2 n-1}=\sum_{k=0}^{2 n-1}\left(\begin{array}{c}
2 n-1 \\
k
\end{array}\right) c^{k} a^{n-k}
$$

by (4.5). Therefore, the formula (4.6) does not contain $1_{Q^{-}}$terms by $(4.4)^{\prime}$.

Note also that, for any $n \in \mathbb{N}$, one has

by (4.5).

$$
\begin{aligned}
l^{2 n} & =\sum_{k=0}^{2 n}\left(\begin{array}{c}
2 n \\
k
\end{array}\right) c^{k} a^{n-k} \\
& =\left(\begin{array}{c}
2 n \\
n
\end{array}\right) c^{n} a^{n}+[\text { Rest terms }],
\end{aligned}
$$

Proposition 2. Let $l$ be the radial operator generating the radial algebra $\mathfrak{L}$ on $Q$. Then,

$$
\begin{aligned}
& l^{2 n-1} \text { does not contain } 1_{Q} \text {-terms in } \mathfrak{L}, \\
& l^{2 n} \text { contains }\left(\begin{array}{c}
2 n \\
n
\end{array}\right) \cdot 1_{Q} \text { in } \mathfrak{L} .
\end{aligned}
$$

Proof. The statement (4.8) (resp., (4.9)) is proven by (4.6) (resp., (4.7)) with help of (4.4), (4.4)' and (4.5).

By (4.1) and (4.2), one can obtain that

$$
\varphi_{j}\left(u_{j}^{2 n-1}\right)=\psi_{j}\left(l^{2 n-1}\left(q_{j}\right)\right)=0,
$$

for all $n \in \mathbb{N}$, by (3.9) and (4.8). Indeed, $l^{2 n-1}\left(q_{j}\right)$ does not contain $q_{j}$-terms by (4.8). Therefore, the formula (4.10) holds.

Similarly, we have

by (4.7)

$$
\varphi_{j}\left(u_{j}^{2 n}\right)=\psi_{j}\left(l^{2 n}\left(q_{j}\right)\right)=\psi_{j}\left(\left(\begin{array}{c}
2 n \\
n
\end{array}\right) q_{j}+[\text { Rest teimrs }]\left(q_{j}\right)\right)
$$

by (3.9), for all $n \in \mathbb{N}$.

$$
=\left(\begin{array}{c}
2 n \\
n
\end{array}\right) \psi_{j}\left(q_{j}\right)=\left(\begin{array}{c}
2 n \\
n
\end{array}\right) \psi\left(q_{j}\right)
$$


Theorem 1. Fix $j \in \mathbb{Z}$, and let $u_{j}=l \otimes q_{j}$ be the corresponding generating operator of the $j$-th probability $\operatorname{space}\left(\mathfrak{L}_{Q}, \varphi_{j}\right)$. Then,

$$
\varphi_{j}\left(u_{j}^{n}\right)=\omega_{n}\left(\left(\frac{n}{2}+1\right) \psi\left(q_{j}\right)\right) c_{\frac{n}{2}},
$$

where $\omega_{n}$ are in the sense of (3.3), and $c_{\frac{n}{2}}$ are the $\left(\frac{n}{2}\right)$-th Catalan numbers, for all $n \in \mathbb{N}$.

Proof. Observe that

$$
\varphi_{j}\left(u_{j}^{2 n-1}\right)=0, \text { for all } n \in \mathbb{N},
$$

by (4.10). In addition, one has that

$$
\begin{aligned}
\varphi_{j}\left(u_{j}^{2 n}\right) & =\left(\begin{array}{c}
2 n \\
n
\end{array}\right) \psi\left(q_{j}\right)=\left(\frac{n+1}{n+1}\right)\left(\begin{array}{c}
2 n \\
n
\end{array}\right) \psi\left(q_{j}\right) \\
& =\left((n+1) \psi\left(q_{j}\right)\right)\left(\frac{1}{n+1}\left(\begin{array}{c}
2 n \\
n
\end{array}\right)\right) \\
& =\left((n+1) \psi\left(q_{j}\right)\right) c_{n},
\end{aligned}
$$

by (4.11), for all $n \in \mathbb{N}$.

Motivated by the free-distributional data (4.12) of the generating operator $u_{j}=l \otimes q_{j}$ of the radial projection algebra $\mathfrak{L}_{Q}$ of (3.14), we define the following morphism

$$
E_{j, Q}: \mathfrak{L}_{Q} \rightarrow \mathfrak{L}_{Q}
$$

by a linear transformation satisfying that

$$
E_{j, Q}\left(u_{i}^{n}\right) \stackrel{\text { def }}{=} \begin{cases}\frac{\psi\left(q_{j}\right)^{n-1}}{\left(\left[\frac{n}{2}\right]+1\right)} u_{j}^{n} & \text { if } i=j, \\ 0_{\mathfrak{L}_{Q^{\prime}}} \text { the zero operator of } \mathfrak{L}_{Q} & \text { otherwise, }\end{cases}
$$

for all $n \in \mathbb{N}, i, j \in \mathbb{Z}$, where $\left[\frac{n}{2}\right]$ means the minimal integer greater than or equal to $\frac{n}{2}$, for example,

$$
\left[\frac{3}{2}\right]=2=\left[\frac{4}{2}\right] .
$$

The linear transformations $E_{j, Q}$ of (4.13) are well-defined linear transformations on $\mathfrak{L}_{Q}$ because of the construction (3.14) of $\mathfrak{L}_{Q}=\mathfrak{L} \otimes_{\mathbb{C}} Q$, and by the structure theorem (3.8) of the radial algebra $\mathfrak{L}$.

Define now a new linear functional $\tau_{j}$ on $\mathfrak{L}_{Q}$ by

$$
\tau_{j} \stackrel{\text { def }}{=} \varphi_{j} \circ E_{j, Q} \text { on } \mathfrak{L}_{Q}, \text { for all } j \in \mathbb{Z},
$$

where $\varphi_{j}$ are in the sense of (4.2).

By the linearity of $\varphi_{j}$ and $E_{j, Q}$, the above morphisms $\tau_{j}$ are indeed well-defined linear functionals on $\mathfrak{L}_{Q}$, for all $j \in \mathbb{Z}$.

Definition 8. The well-defined Banach $*$-probability spaces

$$
\mathfrak{L}_{Q}(j) \stackrel{\text { denote }}{=}\left(\mathfrak{L}_{Q}, \tau_{j}\right)
$$


are called the $j$-th filtered (Banach-*-)probability spaces of the radial projection algebra $\mathfrak{L}_{Q}$ on $Q$, for all $j \in \mathbb{Z}$.

On the $j$-th filtered probability space $\mathfrak{L}_{Q}(j)$ of $(4.15)$, one can obtain that

$$
\begin{aligned}
\tau_{j}\left(u_{j}^{n}\right) & =\varphi_{j}\left(E_{j, Q}\left(u_{j}^{n}\right)\right) \\
& =\varphi_{j}\left(\frac{\psi\left(q_{j}\right)^{n-1}}{\left(\left[\frac{n}{2}\right]+1\right)}\left(u_{j}^{n}\right)\right)=\frac{\psi\left(q_{j}\right)^{n-1}}{\left(\left[\frac{n}{2}\right]+1\right)} \varphi_{j}\left(u_{j}^{n}\right) \\
& =\frac{\psi\left(q_{j}\right)^{n-1}}{\left(\left[\frac{n}{2}\right]+1\right)} \omega_{n}\left(\left(\frac{n}{2}+1\right) \psi\left(q_{j}\right)\right) c_{\frac{n}{2}},
\end{aligned}
$$

i.e., we can get that

for all $n \in \mathbb{N}$, for $j \in \mathbb{Z}$, by (4.12).

$$
\tau_{j}\left(u_{j}^{n}\right)=\omega_{n} \psi\left(q_{j}\right)^{n} c_{\frac{n}{2}}
$$

Theorem 2. Let $\mathfrak{L}_{Q}(j)=\left(\mathfrak{L}_{Q}, \tau_{j}\right)$ be the $j$-th filtered probability space of the radial projection algebra $\mathfrak{L}_{Q}$ on $Q$, for an arbitrarily fixed $j \in \mathbb{Z}$. Then,

$$
\tau_{j}\left(u_{i}^{n}\right)=\delta_{i, j}\left(\omega_{n} \psi\left(q_{j}\right)^{n} c_{\frac{n}{2}}\right)
$$

for all $n \in \mathbb{N}$, for all $i \in \mathbb{Z}$, where $\omega_{n}$ are in the sense of (3.3).

Proof. If $i=j$ in $\mathbb{Z}$, then the free momental data (4.17) holds true by (4.16), for all $n \in \mathbb{N}$.

If $i \neq j$ in $\mathbb{Z}$, then, by the very definition (4.13) of the $j$-th filterization $E_{j, Q}$, and also by the definition (4.2) of $\varphi_{j}$,

$$
\tau_{j}\left(u_{i}^{n}\right)=0, \text { for all } n \in \mathbb{N} .
$$

Therefore, the above formula (4.17) holds, for all $i \in \mathbb{Z}$.

The following corollary is a direct consequence of the above free distribution (4.17).

Corollary 1. Let $\mathfrak{L}_{Q}(j)$ be the $j$-th filtered probability space of $\mathfrak{L}_{Q}$, for a fixed $j \in \mathbb{Z}$, and let $u_{j}=l \otimes q_{j}$ be the $j$-th generating operator of $\mathfrak{L}_{Q}$. Then, $u_{j}$ is $\psi\left(q_{j}\right)^{2}$-semicircular in $\mathfrak{L}_{Q}(j)$.

Proof. First, remark that the $j$-th generating operator $u_{j}$ of $\mathfrak{L}_{Q}(j)$ is self-adjoint in $\mathfrak{L}_{Q}$ because

$$
u_{j}^{*}=\left(l \otimes q_{j}\right)^{*}=l^{*} \otimes q_{j}^{*}=l \otimes q_{j}=u_{j} .
$$

The $\psi\left(q_{j}\right)^{2}$-semicircularity of $u_{j}$ is proven by the above self-adjointness, the free-moment computation (4.17), and the weighted-semicircularity characterization (3.3).

Readers can check that the $j$-th generating operator $u_{j}$ satisfies the free-cumulant formula

$$
k_{n}^{j}\left(u_{j}, \ldots, u_{j}\right)= \begin{cases}\psi\left(q_{j}\right)^{2} & \text { if } n=2, \\ 0 & \text { otherwise }\end{cases}
$$

for all $n \in \mathbb{N}$, by the Möbius inversion of [9], where $k_{n}^{j}(\ldots)$ is the free cumulant on $\mathfrak{L}_{Q}$ in terms of $\tau_{j}$, for all $j \in \mathbb{Z}$. Thus, by the definition (3.1), the free random variables $u_{j}$ are $\psi\left(q_{j}\right)^{2}$-semicircular in the $j$-th filtered probability spaces $\mathfrak{L}_{Q}(j)=\left(\mathfrak{L}_{Q}, \tau_{j}\right)$, for all $j \in \mathbb{Z}$.

Remark that, the $k$-th generating operators $u_{k}$ of the $j$-th filtered probability space $\mathfrak{L}_{Q}(j)$ have zero-free distributions, whenever $k \neq j$ in $\mathbb{Z}$, also, by (4.17). Therefore, in summary, we have the following theorem. 
Theorem 3. Let $u_{k}=l \otimes q_{k}$ be the generating operators of the $j$-th filtered probability space $\mathfrak{L}_{Q}(j)$, for all $k \in \mathbb{Z}$, for a fixed $j \in \mathbb{Z}$. Then,

$$
\text { the } j \text {-th generating operator } u_{j} \text { is } \psi\left(q_{j}\right)^{2} \text {-semicircular in } \mathfrak{L}_{Q}(j) \text {, }
$$

the $k$-th generating operators $u_{k}$ have zero-free distributions, for all $k \neq j$ in $\mathbb{Z}$.

Proof. The proof of the statement (4.19) is done by (4.17) and (4.18). The statement (4.20) is also shown by (4.17). Indeed, if $k \neq j$ in $\mathbb{Z}$, then

$$
\tau_{j}\left(u_{k}^{n}\right)=0, \text { for all } n \in \mathbb{N},
$$

by (4.17). Thus, the free distributions of these self-adjoint operators $u_{k}$ of $\mathfrak{L}_{Q}(j)$, where $k \neq j$ in $\mathbb{Z}$, are characterized by the following free-moment sequences:

$$
\left(\tau_{j}\left(u_{k}^{n}\right)\right)_{n=1}^{\infty}=(0,0,0,0, \ldots) .
$$

Therefore, the free distributions of $u_{k}$ are the zero-free distribution in $\mathfrak{L}_{Q}(j)$, whenever $k \neq j$ in $\mathbb{Z}$.

The above two statements (4.19) and (4.20) fully characterize the free distributions of all generating operators $u_{k}$ of the $j$-th filtered probability spaces $\mathfrak{L}_{Q}(j)$, for all $k, j \in \mathbb{Z}$.

\section{Semicircular Elements Induced by $Q$}

As in Section 4, we keep working on the $j$-th filtered probability spaces, $\mathfrak{L}_{Q}(j)=\left(\mathfrak{L}_{Q}, \tau_{j}\right)$, for $j \in \mathbb{Z}$. The main results of Section 4 show that, for a fixed $j \in \mathbb{Z}$, the $j$-th generating operator $u_{j}=l \otimes q_{j}$ of $\mathfrak{L}_{Q}$ is $\psi\left(q_{j}\right)^{2}$-semicircular in $\mathfrak{L}_{Q}(j)$, by (4.19) (and (4.20)), satisfying that

$$
\tau_{j}\left(u_{j}^{n}\right)=\omega_{n} \psi\left(q_{j}\right)^{n} c_{\frac{n}{2}}
$$

equivalently,

$$
k_{n}^{j}\left(u_{j}, \ldots, u_{j}\right)= \begin{cases}\psi\left(q_{j}\right)^{2} & \text { if } n=2 \\ 0 & \text { otherwise }\end{cases}
$$

for all $n \in \mathbb{N}$.

Recall now that we assumed for convenience that

$$
\psi\left(q_{j}\right) \in \mathbb{C}^{\times}=\mathbb{C} \backslash\{0\}, \text { for all } j \in \mathbb{Z},
$$

in Section 3.

Under our assumption, the generating operators $u_{k}$ of the projection-radial algebra $\mathfrak{L}_{Q}$ induce the operators $U_{k}$

for all $k, j \in \mathbb{Z}$.

$$
U_{k}=\frac{1}{\psi\left(q_{k}\right)} u_{k} \in \mathfrak{L}_{Q}(j)
$$

Theorem 4. Let $U_{k}=\frac{1}{\psi\left(q_{k}\right)} u_{k}$ be free random variables (5.2) of the $j$-th filtered probability space $\mathfrak{L}_{Q}(j)$, for all $k \in \mathbb{Z}$, for a fixed $j \in \mathbb{Z}$.

$$
\text { If } \psi\left(q_{j}\right) \in \mathbb{R}^{\times}=\mathbb{R} \backslash\{0\} \text { in } \mathbb{C} \text {, then } U_{j} \text { is semicircular in } \mathfrak{L}_{Q}(j) .
$$

The operators $U_{k}$ have zero-free distributions in $\mathfrak{L}_{Q}(j)$, whenever $k \neq j$ in $\mathbb{Z}$. 
Proof. Note first that the $k$-th generating operators $u_{k}$ have zero-free distributions in the $j$-th filtered probability space $\mathfrak{L}_{Q}(j)$, whenever $k \neq j$ in $\mathbb{Z}$, by (4.20). Since the corresponding operators $U_{k}$ of $(5.2)$ are the scalar-multiplies of $u_{k}$, if $k \neq j$ in $\mathbb{Z}$, then the operators $U_{k}$ also have zero-free distributions in $\mathfrak{L}_{Q}(j)$. It shows that the statement (5.4) holds.

Assume now that $U_{j}$ is in the sense of (5.2) in $\mathfrak{L}_{Q}(j)$, for $j \in \mathbb{Z}$, and suppose $\psi\left(q_{j}\right) \in \mathbb{R}^{\times}$in $\mathbb{C}$. Since $\psi\left(q_{j}\right) \in \mathbb{R}^{\times}$, the corresponding operator $U_{j}$ is not only well-defined in $\mathfrak{L}_{Q}$, but also self-adjoint in $\mathfrak{L}_{Q}(j)$ by the self-adjointness of $u_{j}$. Therefore, this operator $U_{j}$ is self-adjoint in $\mathfrak{L}_{Q}(j)$, if $\psi\left(q_{j}\right) \in \mathbb{R}^{\times}$.

Under self-adjointness of $U_{j}$, observe that

$$
\begin{aligned}
\tau_{j}\left(U_{j}^{n}\right) & =\tau_{j}\left(\frac{1}{\psi\left(q_{j}\right)^{n}} u_{j}^{n}\right)=\frac{1}{\psi\left(q_{j}\right)^{n}} \tau_{j}\left(u_{j}^{n}\right) \\
& =\frac{1}{\psi\left(q_{j}\right)^{n}}\left(\omega_{n} \psi\left(q_{j}\right)^{n} c_{\frac{n}{2}}\right)=\omega_{n} c_{\frac{n}{2}}
\end{aligned}
$$

by the $\psi\left(q_{j}\right)^{2}$-semicircularity (5.1) of $u_{j}$, for all $n \in \mathbb{N}$.

Therefore, by the semicircularity characterization (3.4), this operator $U_{j}$ is semicircular in $\mathfrak{L}_{Q}(j)$, whenever $\psi\left(q_{j}\right) \in \mathbb{R}^{\times}$. Therefore, the statement (5.3) holds.

The above theorem shows that the operators $U_{j}$ of (5.2), generated by our $\psi\left(q_{j}\right)^{2}$-semicircular elements $u_{j}$, are semicircular in the $j$-th filtered probability spaces $\mathfrak{L}_{Q}(j)$, for all $j \in \mathbb{Z}$, whenever $\psi\left(q_{j}\right) \in \mathbb{R}^{\times}$.

Assumption 5.1 (in short, A 5.1, from below) If there is no confusion, then we automatically assume

$$
\psi\left(q_{j}\right) \in \mathbb{R}^{\times} \text {in } \mathbb{C}, \text { for all } j \in \mathbb{Z},
$$

for all $q_{j} \in \mathbf{Q}$.

The above assumption, A 5.1, will guarantee that, if we have the $\psi\left(q_{j}\right)^{2}$-semicircular elements $u_{j}$ in the $j$-th filtered probability space $\mathfrak{L}_{Q}(j)$, we also have the corresponding semicircular element $U_{j}=\frac{1}{\psi\left(q_{j}\right)} u_{j}$ in $\mathfrak{L}_{Q}(j)$ for all $j \in \mathbb{Z}$.

\section{The Free Product Banach $*$-Probability Space $\underset{j \in \mathbb{Z}}{\star} \underset{Q}{\mathfrak{L}_{Q}}(j)$}

A family $\left\{a_{n}\right\}_{n \in \Lambda}$ in an arbitrary (topological or pure-algebraic) free probability space $(B, \varphi)$ is said to be a free family, if all elements $a_{n}$ of the family are mutually free from each other in $(B, \varphi)$, where $\Lambda$ is a countable (finite or infinite) index set. For such a free family $\left\{a_{n}\right\}_{n \in \Lambda}$, if every element $a_{n}$ is weighted-semicircular (or semicircular), then we call the free family, free weighted-semicircular (respectively, semicircular) family in $(\boldsymbol{B}, \boldsymbol{\varphi})$.

Recall that, for a fixed $C^{*}$-probability space $(A, \psi)$, if there exists a mutually-orthogonal projections $\left\{q_{j}\right\}_{j \in \mathbb{Z}}$, then one can construct $\psi\left(q_{j}\right)^{2}$-semicircular elements $\boldsymbol{u}_{j}=\boldsymbol{l} \otimes \boldsymbol{q}_{j}$ in the $j$-th filtered probability spaces $\mathfrak{L}_{Q}(j)=\left(\mathfrak{L}_{Q}, \boldsymbol{\tau}_{j}\right)$, for all $j \in \mathbb{Z}$, with

$$
\tau_{j}\left(u_{j}^{n}\right)=\omega_{n}\left(\psi\left(q_{j}\right)\right)^{n} c_{\frac{n}{2}},
$$

where $\omega_{n}$ are in the sense of (3.3), equivalently,

$$
k_{n}^{j}\left(u_{j}, \ldots, u_{j}\right)= \begin{cases}\psi\left(q_{j}\right)^{2} & \text { if } n=2, \\ 0 & \text { otherwise }\end{cases}
$$

for all $n \in \mathbb{N}$, for all $j \in \mathbb{Z}$, by (4.16) and (4.17).

Moreover, one can construct corresponding semicircular elements 


$$
u_{j}=\frac{1}{\psi\left(q_{j}\right)} u_{j} \text { in } \mathfrak{L}_{Q}(j),
$$

for all $j \in \mathbb{Z}$, by (5.3), under A 5.1.

Now, we will construct the free product Banach $*$-probability space $\left(\mathfrak{L}_{Q}(\mathbb{Z}), \tau\right)$, by

$$
\begin{aligned}
\left(\mathfrak{L}_{Q}(\mathbb{Z}), \tau\right) & \stackrel{\text { def }}{=} \underset{j \in \mathbb{Z}}{\star \mathfrak{L}_{Q}(j)} \\
& =\left(\underset{j \in \mathbb{Z}}{\star} \mathfrak{L}_{Q}, \underset{j \in \mathbb{Z}}{\star} \tau_{j}\right),
\end{aligned}
$$

satisfying

$$
\mathfrak{L}_{Q}(\mathbb{Z})=\underset{j \in \mathbb{Z}}{\star} \mathfrak{L}_{Q} \stackrel{\text { *-iso }}{=}\left(\mathfrak{L}_{Q}\right)^{\star|\mathbb{Z}|} \text {, and } \tau=\underset{j \in \mathbb{Z}}{\star} \boldsymbol{\tau}_{j} \text {, }
$$

where $(\star)$ means free product (over $\mathbb{C}$ ) in the sense of $[3,9]$.

Note that the free product of $[3,9]$ is different from a pure-algebraic free product. It is totally depending on given linear functionals.

Definition 9. The free product Banach $*$-probability space $\left(\mathfrak{L}_{Q}(\mathbb{Z}), \tau\right)$ of $(6.3)$ is called the free filterization of $Q$. Sometimes, the Banach $*$-algebra $\mathfrak{L}_{Q}(\mathbb{Z})$ is also said to be the free filterization of $Q$.

By the very construction (6.3) of the free filterization $\left(\mathfrak{L}_{Q}(\mathbb{Z}), \tau\right)$ of $Q$, we obtain the following proposition immediately.

Proposition 3. Let $\left(\mathfrak{L}_{Q}(\mathbb{Z}), \tau\right)$ be the free filterization (6.3), and let $\boldsymbol{u}_{j}$ and $\boldsymbol{U}_{j}$ be in the sense of (6.1) and (6.2), respectively, for all $j \in \mathbb{Z}$.

The family $\left\{\boldsymbol{u}_{j} \in \mathfrak{L}_{Q}(j)\right\}_{j \in \mathbb{Z}}$ is a free weighted-semicircular family in $\mathfrak{L}_{Q}(\mathbb{Z})$.

The family $\left\{U_{j} \in \mathfrak{L}_{Q}(j)\right\}_{j \in \mathbb{Z}}$ is a free semicircular family in $\mathfrak{L}_{Q}(\mathbb{Z})$, under A 5.1.

Proof. By the very definition (6.3) of free filterizations, $u_{j}$ s are free from each other in $\left(\mathfrak{L}_{Q}(\mathbb{Z}), \tau\right)$, for all $j \in \mathbb{Z}$. Indeed, each $\boldsymbol{u}_{j}$ is taken from the free block $\mathfrak{L}_{Q}(j)$ of $\left(\mathfrak{L}_{Q}(\mathbb{Z}), \tau\right)$. Therefore, the family $\left\{u_{j} \in \mathfrak{L}_{Q}(j)\right\}_{j \in \mathbb{Z}}$ forms a free family in $\mathfrak{L}_{Q}(\mathbb{Z})$. Since each $\boldsymbol{u}_{j}$ is $\psi\left(\boldsymbol{q}_{j}\right)^{2}$-semicircular in $\mathfrak{L}_{Q}(j)$, it is $\psi\left(q_{j}\right)^{2}$-semicircular in $\left(\mathfrak{L}_{Q}(\mathbb{Z}) \tau\right)$ because

$$
\tau\left(u_{j}^{n}\right)=\tau_{j}\left(u_{j}^{n}\right)=\omega_{n} \psi\left(q_{j}\right)^{n} c_{\frac{n}{2}},
$$

for all $n \in \mathbb{N}$, for all $j \in \mathbb{Z}$. Thus, this family is a free weighted-semicircular family in $\mathfrak{L}_{Q}(\mathbb{Z})$. Therefore, statement (6.4) holds.

Similarly, one can conclude the family $\left\{U_{j} \in \mathfrak{L}_{Q}(j)\right\}_{j \in \mathbb{Z}}$ is a free semicircular family in $\mathfrak{L}_{Q}(\mathbb{Z})$, showing that the statement (6.5) holds.

\section{Weighted-Semicircularity on Affiliated Free Filterizations}

Let $(A, \psi)$ be a fixed $C^{*}$-probability space, and let $\mathrm{Q}=\left\{q_{j}\right\}_{j \in \mathbb{Z}}$ be a family in $A$, consisting of all mutually orthogonal projections. Let $Q$ be the $C^{*}$-subalgebra $C^{*}(\mathbf{Q})$ of $A$ generated by $Q$, and $\mathfrak{L}_{Q}$, the corresponding radial projection algebra on $Q$, inducing the corresponding $j$-th filtered probability spaces $\mathfrak{L}_{Q}(j)=\left(\mathfrak{L}_{Q}, \boldsymbol{\tau}_{j}\right)$, for all $j \in \mathbb{Z}$. Remember that, by A 5.1,

$$
\psi\left(q_{j}\right) \in \mathbb{R}^{\times} \text {in } \mathbb{C}, \text { for all } j \in \mathbb{Z} .
$$




$$
\mathfrak{L}_{Q}(\mathbb{Z}) \stackrel{\text { denote }}{=}\left(\mathfrak{L}_{Q}(\mathbb{Z}), \tau\right) \text { of } Q,
$$

be the free filterization (6.3), and let

$$
\mathcal{W}_{S} \stackrel{\text { def }}{=}\left\{u_{j} \in \mathfrak{L}_{Q}(j)\right\}_{j \in \mathbb{Z}}
$$

and

$$
\mathcal{S} \stackrel{\text { def }}{=}\left\{u_{j}=\frac{1}{\psi\left(q_{j}\right)} u_{j} \in \mathbb{Z}_{Q}(j)\right\}_{j \in \mathbb{Z}}
$$

be the free weighted-semicircular family (6.4), respectively, the free semicircular family (6.5).

Now, we fix $j \in \mathbb{Z}$, and focus on the free block $\mathfrak{L}_{Q}(j)=\left(\mathfrak{L}_{Q}, \tau_{j}\right)$ of the free filterization $\mathfrak{L}_{Q}(\mathbb{Z})$. In addition, consider the compressed $C^{*}$-subalgebra $A_{j}$,

$$
A_{j} \stackrel{\text { def }}{=} q_{j} A q_{j}, \text { for all } j \in \mathbb{Z},
$$

be $C^{*}$-subalgebras of $A$.

Remark 2. Remark that if the $C^{*}$-algebra $A$ is $*$-isomorphic to $Q=C^{*}(\mathrm{Q})$, then each $C^{*}$-subalgebra $A_{j}$ of (7.2) is $*$-isomorphic to $\mathbb{C} \cdot \boldsymbol{q}_{j}$, for $j \in \mathbb{Z}$, which is not so interesting. However, if $\boldsymbol{A}$ is $*$-isomorphic to $\boldsymbol{M} \otimes_{\mathbb{C}} \boldsymbol{Q}$, for a certain non-trivial $\boldsymbol{C}^{*}$-algebra $\boldsymbol{M}$, then every $\boldsymbol{C}^{*}$-subalgebra $\boldsymbol{A}_{j}$ of (7.2) is $*$-isomorphic to $\boldsymbol{M} \cdot \boldsymbol{q}_{j}=\boldsymbol{M}$, for $j \in \mathbb{Z}$, which are interested.

Motivated by the above remark, we now fix an arbitrary unital tracial $W^{*}$-probability space $(M, t r)$, consisting of the von Neumann algebra $M$, and a bounded linear functional $\operatorname{tr}$ on $M$; i.e.,

$$
\operatorname{tr}\left(\mathbf{1}_{M}\right)=\mathbf{1} \text {, for the identity operator } \mathbf{1}_{M} \text { of } M,
$$

and

$$
\operatorname{tr}\left(m_{1} m_{2}\right)=\operatorname{tr}\left(m_{2} m_{1}\right), \text { for all } m_{1}, m_{2} \in M
$$

Remark 3. There are no typical reasons why we take a unital tracial $\boldsymbol{W}^{*}$-probability space $(\boldsymbol{M}, \boldsymbol{t r})$. One may / can regard $(\boldsymbol{M}, \boldsymbol{t r})$ as a unital tracial $\boldsymbol{C}^{*}$-probability space. However, on the von Neumann algebra $\boldsymbol{M}$, trace-depending operator theory, and operator algebra theory work well, and have been widely studied (as in $\mathbf{I I}_{\mathbf{1}}$, $I I_{\infty}, I I I_{\lambda}$-factor theories, etc.), and such structures have lots of interesting applications not only in operator theory but also in related science fields like quantum physics (under $\boldsymbol{W}^{*}$-topological settings).

One of the possible reasons would be from the main results of [1]. We want to mimic the constructions, and apply the main results of [1] here, as applications of our results in Sections 4-7. In addition, we want to allow a variety of topological settings in our Banach *-probability structures, as generalizations of the results in previous sections.

Now, for our $j$-th filtered probability space $\mathfrak{L}_{Q}(j)=\left(\mathfrak{L}_{Q}, \tau_{j}\right)$, a free block of the free filterization $\mathfrak{L}_{Q}(\mathbb{Z})$, for $j \in \mathbb{Z}$, construct the tensor product Banach $*$-algebra,

$$
\mathfrak{L}_{Q}^{M} \stackrel{\text { def }}{=} M \otimes_{\mathbb{C}} \mathfrak{L}_{Q}
$$

and define a linear functional $\tau_{j}^{M}$ on $\mathfrak{L}_{Q}^{M}$, by a linear morphism,

$$
\tau_{j}^{M} \stackrel{\text { def }}{=} \operatorname{tr} \otimes \tau_{j} \text { on } \mathfrak{L}_{Q}^{M},
$$

in the sense that 


$$
\begin{aligned}
\tau_{j}^{M}\left(m \otimes u_{j}^{n}\right) & \stackrel{\text { def }}{=} \tau_{j}\left(\operatorname{tr}(m) u_{j}^{n}\right) \\
& =\operatorname{tr}(m) \tau_{j}\left(u_{j}^{n}\right),
\end{aligned}
$$

for all $n \in \mathbb{N}_{0}$, for all $m \in(M, t r)$, for all generators $u_{j}=l \otimes q_{j}$ of the radial projection algebra $\mathfrak{L}_{Q}$.

Then, one has well-defined Banach $*$-probability spaces $\left(\mathfrak{L}_{Q}^{M}, \tau_{j}^{M}\right)$, for all $j \in \mathbb{Z}$.

Definition 10. The Banach *-algebra $\mathfrak{L}_{Q}^{M}$ of (7.3) is called the $\mathbf{M}$ (-affiliated)-radial projection algebra. The Banach *-probability spaces $\left(\mathfrak{L}_{Q}^{M}, \tau_{j}^{M}\right)$ of the $M$-radial projection algebra $\mathfrak{L}_{Q}^{M}$, and the linear functionals $\boldsymbol{\tau}_{j}^{M}$ of (7.4) are said to be the $\boldsymbol{j}$-th $\boldsymbol{M}$ (-affiliated)-filtered probability spaces, for all $\boldsymbol{j} \in \mathbb{Z}$. For convenience, we denote our $\mathbf{j}$-th $M$-filtered probability spaces $\left(\mathfrak{L}_{Q}^{M}, \tau_{j}^{M}\right)$ by $\mathfrak{L}_{Q}^{M}(j)$, i.e.,

$$
\mathfrak{L}_{Q}^{M}(j) \stackrel{\text { denote }}{=}\left(\mathfrak{L}_{Q}^{M}, \tau_{j}^{M}\right), \text { for all } j \in \mathbb{Z}
$$

Now, let $\mathfrak{L}_{Q}^{M}(j)=\left(\mathfrak{L}_{Q}^{M}, \tau_{j}^{M}\right)$ be our $j$-th $M$-filtered probability space (7.5), for all $j \in \mathbb{Z}$. Construct the free-product Banach $*$-probability space $\left(\mathfrak{L}_{Q}^{M}(\mathbb{Z}), \tau^{M}\right)$ by

$$
\begin{aligned}
& \mathfrak{L}_{Q}^{M}(\mathbb{Z}) \stackrel{\text { denote }}{=}\left(\mathfrak{L}_{Q}^{M}(\mathbb{Z}), \tau^{M}\right) \stackrel{\text { def }}{=} \underset{j \in \mathbb{Z}}{\star} \mathfrak{L}_{Q}^{M}(j) \\
& =\left(\underset{j \in \mathbb{Z}}{\underset{j \in \mathbb{Q}}{\star}} \mathfrak{L}_{Q^{\prime}}^{M} \underset{j \in \mathbb{Z}}{\underset{j}{\star}} \tau_{j}^{M}\right) .
\end{aligned}
$$

Definition 11. The free-product Banach *-probability space

$$
\mathfrak{L}_{Q}^{M}(\mathbb{Z})=\left(\mathfrak{L}_{Q}^{M}(\mathbb{Z}), \tau^{M}\right)
$$

of (7.6) is called the $\boldsymbol{M}$ (-affiliated)-free filterization of $Q=W^{*}(\mathbf{Q})$.

It is not difficult to check that the elements

$$
\mathbf{1}_{M} \otimes u_{j} \in \mathfrak{L}_{Q}^{M}(\mathbb{Z}), \text { for all } j \in \mathbb{Z},
$$

are $\psi\left(q_{j}\right)^{2}$-semicircular elements in the $M$-free filterization $\mathfrak{L}_{Q}^{M}(\mathbb{Z})=\left(\mathfrak{L}_{Q}^{M}(\mathbb{Z}), \tau^{M}\right)$.

Proposition 4. Let $\mathfrak{L}_{Q}^{M}(\mathbb{Z})$ be an $M$-free filterization (7.6) of $Q$, where $(M$, tr) is a fixed unital tracial $W^{*}$-probability space.

Let $\boldsymbol{u}_{j}^{o}=\mathbf{1}_{M} \otimes \boldsymbol{u}_{j} \in \mathfrak{L}_{Q}^{M}(j)$ in $\mathfrak{L}_{Q}^{M}(\mathbb{Z})$, where $\mathbf{1}_{M}$ is the identity element of $\boldsymbol{M}$, and $\boldsymbol{u}_{j}=\boldsymbol{l} \otimes \boldsymbol{q}_{j} \in \mathfrak{L}_{Q}(j)$, for all $j \in \mathbb{Z}$. Then, $\boldsymbol{u}_{j}^{o}$ are $\psi\left(\boldsymbol{q}_{j}\right)^{2}$-semicircular in the $\boldsymbol{M}$-free filterization $\mathfrak{L}_{Q}^{M}(\mathbb{Z})$.

Let $\boldsymbol{U}_{j}^{o}=\mathbf{1}_{M} \otimes \boldsymbol{U}_{j} \in \mathfrak{L}_{Q}^{M}(j)$ in $\mathfrak{L}_{Q}^{M}(\mathbb{Z})$, where $\boldsymbol{U}_{j}=\frac{1}{\psi\left(q_{j}\right)} \boldsymbol{u}_{j} \in \mathfrak{L}_{Q}(j)$, under $A$ 5.1, for all $j \in \mathbb{Z}$. Then, $u_{j}^{o}$ are semicircular in $\mathfrak{L}_{Q}^{M}(\mathbb{Z})$.

Proof. Let $u_{j}^{o}=\mathbf{1}_{M} \otimes u_{j} \in \mathfrak{L}_{Q}^{M}(j)$ in $\mathfrak{L}_{Q}^{M}(\mathbb{Z})$, for $j \in \mathbb{Z}$. Since $\boldsymbol{u}_{j}^{o}$ is contained in the $j$-th block $\mathfrak{L}_{Q}^{M}(j)$ of the $M$-free filterization $\mathfrak{L}_{Q}^{M}(\mathbb{Z})$, one obtains that 


$$
\begin{aligned}
\tau^{M}\left(\left(u_{j}^{o}\right)^{n}\right) & =\tau^{M}\left(1_{M} \otimes u_{j}^{n}\right)=\operatorname{tr}\left(1_{M}\right) \tau\left(u_{j}^{n}\right) \\
& =\operatorname{tr}\left(1_{M}\right) \tau_{j}\left(u_{j}^{n}\right) \\
& =1 \cdot\left(\omega_{n} \psi\left(q_{j}\right)^{n} c \frac{n}{2}\right)=\omega_{n} \psi\left(q_{j}\right)^{n} c_{\frac{n}{2}},
\end{aligned}
$$

by (7.5) and (7.6), for all $\boldsymbol{n} \in \mathbb{N}$. Therefore, by (7.9), (3.1) and (3.3), the free random variables $\boldsymbol{u}_{j}^{\boldsymbol{o}}$ are $\psi\left(q_{j}\right)^{2}$-semicircular in the $M$-free filterization $\mathfrak{L}_{Q}^{M}(\mathbb{Z})$, for all $j \in \mathbb{Z}$, i.e., the statement (7.7) holds.

Similarly, since $U_{j}^{o}$ is in the free block $\mathfrak{L}_{Q}^{M}(j)$ of $\mathfrak{L}_{Q}^{M}(\mathbb{Z})$, one obtains that

$$
\tau^{M}\left(\left(U_{j}^{o}\right)^{n}\right)=\operatorname{tr}\left(\mathbf{1}_{M}\right) \tau_{j}\left(U_{j}^{n}\right)=\omega_{n} c_{\frac{n}{2}},
$$

for all $n \in \mathbb{N}$. Therefore, by (7.10), (3.2) and (3.4), the free random variables $U_{j}^{o}$ are semicircular in $\mathfrak{L}_{Q}^{M}(\mathbb{Z})$.

By (7.7) and (7.8), we obtain the following corollary immediately.

Corollary 2. Let $\mathfrak{L}_{Q}^{M}(\mathbb{Z})$ be $M$-free filterization (7.6) of $Q$ and $(M, t r)$, and suppose $\mathfrak{L}_{Q}^{M}(j)$ are the free blocks (7.5) of $\mathfrak{L}_{Q}^{M}(\mathbb{Z})$, for all $j \in \mathbb{Z}$.

The family $\left\{\mathbf{1}_{M} \otimes u_{j} \in \mathfrak{L}_{Q}^{M}(j)\right\}_{j \in \mathbb{Z}}$ is a free weighted-semicircular family in $\mathfrak{L}_{Q}^{M}(\mathbb{Z})$.

The family $\left\{\mathbf{1}_{M} \otimes \boldsymbol{U}_{j} \in \mathfrak{L}_{Q}^{M}(j)\right\}_{j \in \mathbb{Z}}$ is a free semicircular family in $\mathfrak{L}_{Q}^{M}(\mathbb{Z})$.

Proof. By (7.7) and (7.8), the operators

$$
\boldsymbol{u}_{j}^{o}=\mathbf{1}_{M} \otimes u_{j} \in \mathfrak{L}_{Q}^{M}(j)
$$

are $\psi\left(\boldsymbol{q}_{j}\right)^{2}$-semicircular in the $M$-free filterization $\mathfrak{L}_{Q}^{M}(\mathbb{Z})$, and the operators

$$
U_{j}^{o}=\mathbf{1}_{M} \otimes U_{j} \in \mathfrak{L}_{Q}^{M}(j)
$$

are semicircular in $\mathfrak{L}_{Q}(\mathbb{Z})$, respectively.

Moreover, since all elements $\boldsymbol{u}_{j}^{o}$ (or $\boldsymbol{U}_{j}^{o}$ ) are contained in the mutually-distinct free blocks $\mathfrak{L}_{Q}^{M}(j)$ of $\mathfrak{L}_{Q}^{M}(\mathbb{Z})$, for all $j \in \mathbb{Z}$, the free random variables $\boldsymbol{u}_{j}^{o}$ (resp., $\boldsymbol{U}_{j}^{o}$ ) are mutually free from each other in $\mathfrak{L}_{Q}(\mathbb{Z})$. Therefore, the statements (7.11) and (7.12) hold.

Now, we take

$$
\mathcal{Q} \stackrel{\text { def }}{=}\left\{u_{j}^{o}=\mathbf{1}_{M} \otimes u_{j} \in \mathfrak{L}_{Q}^{M}(j)\right\}_{j \in \mathbb{Z}^{\prime}}
$$

and

$$
\mathcal{X} \stackrel{\text { def }}{=}\left\{u_{j}^{o}=1_{M} \otimes U_{j} \in \mathfrak{L}_{Q}^{M}(j)\right\}_{j \in \mathbb{Z}}
$$

in the $M$-free filterization $\mathfrak{L}_{Q}^{M}(\mathbb{Z})$ of $Q$ and $(M, t r)$.

By (7.11) and (7.12), the family $\mathcal{Q}$ (resp., $\mathcal{X})$ of (7.13) is a free weighted-semicircular (Respectively, semicircular) family in $\mathfrak{L}_{Q}^{M}(\mathbb{Z})$.

From the free families $\mathcal{Q}$ and $\mathcal{X}$ of (7.13), let us construct families,

$$
\left\{m_{j} \otimes u_{j} \in \mathfrak{L}_{Q}^{M}(j)\right\}_{j \in \mathbb{Z}}
$$


and

$$
\left\{m_{j} \otimes U_{j} \in \mathfrak{L}_{Q}^{M}(j)\right\}_{j \in \mathbb{Z}},
$$

where $m_{j}$ are elements of $M$, satisfying

$$
m_{j} \neq \mathbf{0}_{M} \text {, and } m_{j} \neq \mathbf{1}_{M},
$$

where $\mathbf{0}_{M}$ means the zero element of $M$, for all $j \in \mathbb{Z}$.

Note that, by (7.6), the families (7.14) are free families in $\mathfrak{L}_{Q}^{M}(\mathbb{Z})$. Now, consider certain type of free families (7.14).

Theorem 5. Let $m \in(\boldsymbol{M}, \boldsymbol{t r})$ be nonzero, and assume that (i) $\boldsymbol{m}$ is self-adjoint, and (ii) there exists $\boldsymbol{t}_{\mathbf{0}} \in \mathbb{C}^{\times}$, such that

$$
\operatorname{tr}\left(m^{n}\right)=t_{0}^{n} \text {, for all } n \in \mathbb{N} \text {. }
$$

The family $\left\{m \otimes U_{j} \in \mathfrak{L}_{Q}^{M}(j)\right\}_{j \in \mathbb{Z}}$ in the sense of (7.14) is a free $\boldsymbol{t}_{0}^{2}$-semicircular family in $\mathfrak{L}_{Q}^{M}(\mathbb{Z})$.

The family $\left\{m \otimes u_{j} \in \mathfrak{L}_{Q}^{M}(j)\right\}_{j \in \mathbb{Z}}$ in the sense of (7.14) is a free weighted-semicircular family in $\mathfrak{L}_{Q}^{M}(\mathbb{Z})$. In particular, each element $m \otimes u_{j}^{o}$ is $\left(t_{0} \psi\left(q_{j}\right)\right)^{2}$-semicircular in $\mathfrak{L}_{Q}^{M}(\mathbb{Z})$, for all $j \in \mathbb{Z}$.

Proof. For convenience, let us denote the two families of (7.15) and (7.16) by

$$
m \mathcal{X} \text {, respectively, } m \mathcal{Q},
$$

where $\mathcal{Q}$ and $\mathcal{X}$ are in the sense of (7.13), where $m \in(M, t r)$ is given as above.

First of all, by the self-adjointness of $x \in \mathcal{Q} \cup \mathcal{X}$, since $m$ is assumed to be self-adjoint in $M$, all elements

$$
m \otimes u_{j}, m \otimes U_{j} \in m \mathcal{X} \cup m \mathcal{Q}
$$

are self-adjoint in $\mathfrak{L}_{Q}^{M}(\mathbb{Z})$.

All elements $m \otimes U_{j}^{o} \in m \mathcal{X}$ are contained in the mutually-distinct free blocks,

$$
\mathfrak{L}_{Q}^{M}(j)=\left(\mathfrak{L}_{Q}^{M}, \tau_{j}^{M}\right)=\left(M \otimes_{\mathbb{C}} \mathfrak{L}_{Q}, \operatorname{tr} \otimes \tau_{j}\right),
$$

for all $j \in \mathbb{Z}$, these operators $m \otimes U_{j}$ are mutually free from each other in $\mathfrak{L}_{Q}^{M}(\mathbb{Z})$, for all $j \in \mathbb{Z}$.

Observe now that

$$
\begin{gathered}
\tau^{M}\left(\left(m \otimes U_{j}\right)^{n}\right)=\tau^{M}\left(m^{n} \otimes U_{j}^{n}\right)=\tau_{j}^{M}\left(m^{n} \otimes U_{j}^{n}\right) \\
\quad=\operatorname{tr}\left(m^{n}\right) \tau_{j}^{0}\left(U_{j}^{n}\right)=t_{0}^{n} \omega_{n} c_{\frac{n}{2}}=\omega_{n} t_{0}^{n} c_{\frac{n}{2}}
\end{gathered}
$$

for all $n \in \mathbb{N}$, by the assumption that $\operatorname{tr}\left(m^{n}\right)=t_{0}^{n}$, for all $n \in \mathbb{N}$, for some $t_{0} \in \mathbb{C}^{\times}$.

It shows that the self-adjoint free random variables $m \otimes U_{j} \in m \mathcal{X}$ are $t_{0}^{2}$-semicircular in the $M$-free filterization $\mathfrak{L}_{Q}^{M}(\mathbb{Z})$, by (3.3). Therefore, the family $m \mathcal{X}$ is a free $t_{0}^{2}$-semicircular family in $\mathfrak{L}_{Q}^{M}(\mathbb{Z})$.

Similarly, since $u_{j}^{o}=\psi\left(q_{j}\right) U_{j}^{o} \in \mathfrak{L}_{Q}^{M}(j)$ in $\mathfrak{L}_{Q}^{M}(\mathbb{Z})$, for all $j \in \mathbb{Z}$, the family $m \mathcal{Q}$ is a free family in $\mathfrak{L}_{Q}^{M}(\mathbb{Z})$ because $m \mathcal{X}$ is. By A 5.1, and by the condition $m$ is self-adjoint, all entries $\boldsymbol{m} \otimes \boldsymbol{u}_{j}$ of $m \mathcal{Q}$ are self-adjoint in $\mathfrak{L}_{Q}(\mathbb{Z})$.

In addition, one has that 


$$
\begin{aligned}
\tau^{M}\left(\left(m \otimes u_{j}\right)^{n}\right) & =\operatorname{tr}\left(m^{n}\right) \tau_{j}\left(u_{j}^{n}\right) \\
& =t_{0}^{n}\left(\omega_{n} \psi\left(q_{j}\right)^{n} c_{\frac{n}{2}}\right) \\
& =\omega_{n}\left(t_{0} \psi\left(q_{j}\right)\right)^{n} c_{\frac{n}{2}}
\end{aligned}
$$

for all $n \in \mathbb{N}$.

Therefore, by (3.4), each entry $m \otimes \boldsymbol{u}_{j}$ of the family is $\left(\boldsymbol{t}_{0} \psi\left(\boldsymbol{q}_{j}\right)\right)^{2}$-semicircular in $\mathfrak{L}_{Q}^{M}(\mathbb{Z})$, for all $j \in \mathbb{Z}$, and, hence, the family $m \mathcal{Q}$ is a free weighted-semicircular family in the $\boldsymbol{M}$-free filterization $\mathfrak{L}_{Q}^{M}(\mathbb{Z})$.

Let's denote the families

$$
\left\{m_{j} \otimes U_{j} \in \mathfrak{L}_{Q}^{M}(j)\right\}_{j \in \mathbb{Z}} \text {, and }\left\{m_{j} \otimes u_{j} \in \mathfrak{L}_{Q}^{M}(j)\right\}_{j \in \mathbb{Z}}
$$

of (7.14), by

$$
\mathcal{X}_{M} \text {, respectively, } \mathcal{Q}_{M},
$$

for $m_{j} \in(M, t r)$, for all $j \in \mathbb{Z}$.

Under A 5.1, every semicircular element $U_{j}$ (which is a tensor-factor of $m_{j} \otimes U_{j} \in \mathcal{X}_{M}$ ) is well-defined as the scalar-product $\frac{1}{\psi\left(q_{j}\right)} u_{j}$ of the $\psi\left(q_{j}\right)^{2}$-semicircular element $u_{j}$ in the free block $\mathfrak{L}_{Q}(j)$ of the free filterization $\mathfrak{L}_{Q}(\mathbb{Z})$ of $Q$ (which is a tensor-factor of $\mathfrak{L}_{Q}^{M}(\mathbb{Z})$ ), for all $j \in \mathbb{Z}$. Thus, one can understand $u_{j}$ as $\psi\left(\boldsymbol{q}_{j}\right) \boldsymbol{U}_{j}$, and, hence,

$$
\begin{aligned}
m_{j} \otimes u_{j} & =m_{j} \otimes\left(\psi\left(q_{j}\right) U_{j}\right) \\
& =\psi\left(q_{j}\right)\left(m_{j} \otimes U_{j}\right) \\
& =\left(\psi\left(q_{j}\right) m_{j}\right) \otimes U_{j},
\end{aligned}
$$

in $\mathfrak{L}_{Q}^{M}(\mathbb{Z})$, for all $j \in \mathbb{Z}$.

It means that the family $\mathcal{Q}_{M}$ (or $\mathcal{X}_{M}$ ) is generated by the family $\mathcal{X}_{M}$ (resp., $\mathcal{Q}_{M}$ ). Therefore, in the following, we concentrate on studying properties of the operators of $\mathfrak{L}_{Q}^{M}(\mathbb{Z})$ induced by $\mathcal{X}_{M}$ (covering the properties of those induced by $\mathcal{Q}_{M}$ in the above senses).

\section{Free Distributions on Affiliated Free Filterizations}

In this section, we fix a $M$-free filterization $\mathfrak{L}_{Q}^{M}(\mathbb{Z})=\left(\mathfrak{L}_{Q}^{M}(\mathbb{Z}), \tau^{M}\right)$ in the sense of (7.6) for a fixed unital tracial $W^{*}$-probability space $(M, t r)$, and study free-distributional data of certain free random variables of $\mathfrak{L}_{Q}^{M}(\mathbb{Z})$.

Let $\mathcal{X}=\left\{\mathbf{1}_{M} \otimes \boldsymbol{U}_{j} \in \mathfrak{L}_{Q}^{M}(j)\right\}_{j \in \mathbb{Z}}$ be a free semicircular family of (7.12) in $\mathfrak{L}_{Q}^{M}(\mathbb{Z})$, where $\mathfrak{L}_{Q}^{M}(j)$ are the free blocks of $\mathfrak{L}_{Q}^{M}(\mathbb{Z})$, for all $j \in \mathbb{Z}$. Now, we construct free random variables $T$ of $\mathfrak{L}_{Q}^{M}(\mathbb{Z})$ induced by $\boldsymbol{M}$ and $\mathcal{X}$,

$$
T=\sum_{j \in \mathbb{Z}} m_{j} \otimes U_{j}^{k_{j}}
$$

with $m_{j} \in(M, t r)$, and $k_{j} \in \mathbb{N}$, where the summands of (8.1) satisfy

$$
m_{j} \otimes u_{j}^{k_{j}}=\left(m_{j} \otimes 1_{\mathfrak{L}_{Q}(\mathbb{Z})}\right)\left(1_{M} \otimes u_{j}^{k_{j}}\right),
$$

where $\mathbf{1}_{M} \otimes U_{j} \in \mathcal{X}$, for all $j \in \mathbb{Z}$.

For an operator $T$ of (8.1), define the support of $T$, denoted by $\operatorname{Supp}(T)$, by

$$
\operatorname{Supp}(T)=\left\{j \in \mathbb{Z}: m_{j} \neq \mathbf{0}_{M}\right\} \text { in } \mathbb{Z} .
$$

Proposition 5. Let $\mathbf{T}$ be a free random variable (8.1) in the $\boldsymbol{M}$-free filterization $\mathfrak{L}_{Q}^{M}(\mathbb{Z})$. Then, 


$$
\tau^{M}(T)=\sum_{j \in \operatorname{Supp}(T)} \omega_{k_{j}} \operatorname{tr}\left(m_{j}\right) c_{k_{j}}{ }^{\prime}
$$

where $\omega_{k}$ are in the sense of (3.3), for all $k \in \mathbb{N}$, and $\operatorname{Supp}(T)$ is the support (8.2) of $\boldsymbol{T}$ in $\mathbb{Z}$.

Proof. Observe that

by $(8.1)$

$$
\begin{aligned}
\tau^{M}(T) & =\sum_{j \in \mathbb{Z}} \tau^{M}\left(m_{j} \otimes U_{j}^{k_{j}}\right) \\
& =\sum_{j \in \mathbb{Z}} \operatorname{tr}\left(m_{j}\right) \tau\left(u_{j}^{k_{j}}\right)=\sum_{j \in \mathbb{Z}} \operatorname{tr}\left(m_{j}\right) \tau_{j}\left(u_{j}^{k_{j}}\right)
\end{aligned}
$$

by $(8.1)^{\prime}$

$$
=\sum_{j \in \mathbb{Z}} \operatorname{tr}\left(m_{j}\right)\left(\omega_{k_{j}} c_{\frac{k_{j}}{2}}\right)
$$

$$
=\sum_{j \in S u p p(T)} \operatorname{tr}\left(m_{j}\right)\left(\omega_{k_{j}} c_{\frac{k_{j}}{2}}\right) .
$$

Therefore the formula (8.3) holds.

Observe first that if $T$ is in the sense of (8.1) in the $M$-free filterization $\mathfrak{L}_{Q}^{M}(\mathbb{Z})$, then each summand $m_{j} \otimes U_{j}^{k_{j}}$, such that $m_{j} \neq 0_{M}$, equivalently, with $j \in \operatorname{Sup} p(T)$, are contained in a free block $\mathfrak{L}_{Q}^{M}(j)=\left(\mathfrak{L}_{Q}^{M}, \tau_{j}^{M}\right)$, for all $j \in \operatorname{Supp}(T)$, in $\mathfrak{L}_{Q}^{M}(\mathbb{Z})$. Therefore, one can conclude the following result.

Proposition 6. Let $\mathbf{T}$ be in the sense of (8.1) induced by a fixed $\boldsymbol{W}^{*}$-probability space $(\boldsymbol{M}, \boldsymbol{t r})$, and the free semicircular family $\mathcal{X}$ of (7.12) in $\mathfrak{L}_{Q}^{M}(\mathbb{Z})$. Then, all nonzero summands $\boldsymbol{m}_{j} \otimes \boldsymbol{U}_{j}^{n_{j}}$ of $\boldsymbol{T}$ are free from each other in the $M$-free filterization $\mathfrak{L}_{Q}^{M}(\mathbb{Z})$, for all $j \in \operatorname{Supp}(T)$. Equivalently, this operator $T$ is a free sum in $\mathfrak{L}_{Q}^{M}(\mathbb{Z})$.

Proof. The proof is straightforward from the very construction (8.1) of the operator $T$ in $\mathfrak{L}_{Q}^{M}(\mathbb{Z})$, as we discussed in the very above paragraph.

Now, we concentrate on studying the free distribution of a free sums $T$ of (8.1). Consider first that

$$
\begin{aligned}
\tau^{M}\left(T^{n}\right) & =\tau^{M}\left(\left(\sum_{j \in S u p p(T)} m_{j} \otimes U_{j}^{k_{j}}\right)^{n}\right) \\
& =\tau^{M}\left(\sum_{\left(j_{1}, \ldots, j_{n}\right) \in \operatorname{Supp}(T)^{n}}\left(\prod_{l=1}^{n}\left(m_{j_{l}} \otimes U_{j_{l}}^{k_{j_{l}}}\right)\right)\right),
\end{aligned}
$$

where

$$
\operatorname{Supp}(T)^{n}=\underbrace{\operatorname{Supp}(T) \times \cdots \times \operatorname{Supp}(T)}_{n \text {-times }}
$$

the Cartesian product of $n$-copies of $\operatorname{Supp}(T)$ 


$$
\begin{aligned}
& =\sum_{\left(j_{1}, \ldots, j_{n}\right) \in S u p p(T)^{n}} \tau^{M}\left(\prod_{l=1}^{n}\left(m_{j_{l}} \otimes u_{j_{l}}^{k_{j_{l}}}\right)\right) \\
& =\sum_{\left(j_{1}, \ldots, j_{n}\right) \in S u p p(T)^{n}} \tau^{M}\left(\left(\prod_{l=1}^{n} m_{j_{l}}\right) \otimes\left(\prod_{l=1}^{n} u_{j_{l}}^{k_{j_{l}}}\right)\right)
\end{aligned}
$$

by (7.6),

$$
=\sum_{\left(j_{1}, \ldots, j_{n}\right) \in \operatorname{Supp}(T)^{n}} \operatorname{tr}\left(\prod_{l=1}^{n} m_{j_{l}}\right) \tau\left(\prod_{l=1}^{n} u_{j_{l}}^{k_{j_{l}}}\right)
$$

Lemma 1. Let $\mathbf{T}$ be a free sum (8.1) in the $M$-free filterization $\mathfrak{L}_{Q}^{M}(\mathbb{Z})$. Then,

$$
\tau^{M}\left(T^{n}\right)=\sum_{\left(j_{1}, \ldots, j_{n}\right) \in S u p p(T)^{n}} \operatorname{tr}\left(\prod_{l=1}^{n} m_{j_{l}}\right) \tau\left(\prod_{l=1}^{n} U_{j_{l}}^{k_{j_{l}}}\right),
$$

for all $n \in \mathbb{N}$, where $\tau$ is the trace of (6.3) on the free filterization $\mathfrak{L}_{Q}(\mathbb{Z})$ of $Q$.

Proof. The proof of (8.5) is done by (8.4).

The above formula (8.5) shows that computing free moments of the free sum $T$ of (8.1) is reduced to compute the joint free moments of semicircular elements

$$
\left\{u_{j} \in \mathfrak{L}_{Q}(j)\right\}_{j \in \mathbb{Z}}
$$

of the free filterization $\mathfrak{L}_{Q}(\mathbb{Z})$ of $Q$.

Lemma 2. Let $\mathcal{X}_{Q}=\left\{U_{j} \in \mathfrak{L}_{Q}(j)\right\}_{j \in \mathbb{Z}}$ be the free semicircular family (6.5) in the free filterization $\mathfrak{L}_{Q}(\mathbb{Z})$ of $Q$, and let

$$
U=\prod_{l=1}^{n} u_{j_{l}}^{k_{l}} \in \mathfrak{L}_{Q}(\mathbb{Z}), \text { for } k_{l} \in \mathbb{N},
$$

where $j_{1}, \ldots, j_{n} \in \mathbb{Z}$, for $n \in \mathbb{N}$.

$$
\text { If } j_{1}=\ldots=j_{n}=j \text { in } \mathbb{Z}, \text { then the operator } \boldsymbol{U} \text { of (8.6) satisfies that }
$$

$$
\tau(U)=\tau\left(U^{*}\right)=\omega_{N} c_{\frac{N}{2}}, \text { with } N=\sum_{l=1}^{n} k_{l} \text { in } \mathbb{N}
$$

If the sequence $\left(j_{1}, \ldots, j_{n}\right)$ is alternating in $\mathbb{Z}$, in the sense that

$$
j_{1} \neq j_{2}, j_{2} \neq j_{3}, \ldots, j_{n-1} \neq j_{n} \text { in } \mathbb{Z},
$$

then

$$
\tau(U)=\tau\left(U^{*}\right)=\prod_{l=1}^{n} \omega_{n_{l}} c \frac{n_{l}}{2} .
$$

Proof. Let $U$ be an operator (8.6) in the free filterization $\mathfrak{L}_{Q}(\mathbb{Z})$ of $Q$. If $j_{1}=\ldots=j_{n}=j$ in $\mathbb{Z}$, then

$$
\tau(U)=\tau\left(U_{j}^{k_{1}} \ldots U_{j}^{k_{n}}\right)=\tau\left(U_{j}^{N}\right)
$$

with $N=\sum_{l=1}^{n} k_{l}$ in $\mathbb{N}$ 


$$
=\tau_{j}\left(U_{j}^{N}\right)=\omega_{N} c_{\frac{N}{2}},
$$

by the semicircularity (6.5) of $U_{j}$ in $\mathfrak{L}_{Q}(\mathbb{Z})$.

Similarly, by the self-adjointness of $\boldsymbol{U}_{j}$, one can get that

$$
u^{*}=\left(u_{j}^{k_{1}} \ldots u_{j}^{k_{n}}\right)^{*}=u_{j}^{k_{n}} u_{j}^{k_{n-1}} \ldots u_{j}^{k_{1}} \text { in } \mathfrak{L}_{Q}(\mathbb{Z}),
$$

and, hence,

$$
\tau\left(U^{*}\right)=\tau\left(U_{j}^{k_{n}} U_{j}^{k_{n-1}} \ldots U_{j}^{k_{1}}\right)=\tau\left(U_{j}^{N}\right)=\omega_{N} c_{\frac{N}{2}},
$$

with $N=\sum_{l=1}^{n} k_{l}$ as above in $\mathbb{N}$.

Thus, the statement (8.7) holds.

Assume now that the sequence $\left(j_{1}, \ldots, j_{n}\right)$ is alternating in $\mathbb{Z}$. Then, by the freeness (6.5) of the family $\mathcal{X}_{Q}$ in $\mathfrak{L}_{Q}(\mathbb{Z})$, one obtains that

$$
\tau(U)=\tau\left(\prod_{l=1}^{n} U_{j_{l}}^{n_{l}}\right)=\prod_{l=1}^{n} \tau_{j_{l}}\left(U_{j_{l}}^{n_{l}}\right)=\prod_{l=1}^{n} \omega_{n_{l}} c_{\frac{n_{l}}{2}},
$$

by the semicircularity on $\mathcal{X}_{Q}$ in $\mathfrak{L}_{Q}(\mathbb{Z})$. In addition, one can get that

$$
\tau\left(U^{*}\right)=\tau\left(\prod_{l=1}^{n} U_{j_{n-l+1}}^{n_{l-n+1}}\right)=\prod_{l=1}^{n} \tau_{j_{n-l+1}}\left(U_{j_{n-l+1}}^{n_{l-n+1}}\right)=\tau(U) .
$$

Therefore, the statement (8.8) holds.

The above results (8.7) and (8.8) in fact characterize the free distributions of the product operators of $\mathfrak{L}_{Q}(\mathbb{Z})$ in $\mathcal{X}_{Q}$ because of the freeness on the free semicircular family $\mathcal{X}_{Q}$. Indeed, every product $T$ in $\mathcal{X}_{Q}$ has its unique form,

$$
T=\prod_{l=1}^{n} U_{j_{l}}^{n_{l}} \text { in } \mathfrak{L}_{Q}(\mathbb{Z}),
$$

where $\left(j_{1}, \ldots, j_{n}\right)$ is alternating in $\mathbb{Z}$. The resulted unique forms under product are said to be the free reduced words of $\mathfrak{L}_{Q}(\mathbb{Z})$ in $\mathcal{X}_{Q}$.

For instance, if $X$ is a product,

$$
X=U_{-1} U_{-1} U_{0} U_{-1} U_{4} U_{4} U_{4} \in \mathfrak{L}_{Q}(\mathbb{Z}) \text { in } \mathcal{X}_{Q},
$$

then it is in fact

$$
X=U_{-1}^{2} U_{0} U_{-1} U_{4}^{3} \text { in } \mathfrak{L}_{Q}(\mathbb{Z}),
$$

satisfying

$$
\begin{gathered}
U_{-1}^{2} \in \mathfrak{L}_{Q}(-1), U_{0} \in \mathfrak{L}_{Q}(0), \\
U_{-1} \in \mathfrak{L}_{Q}(-1), \text { and } U_{4}^{3} \in \mathfrak{L}_{Q}(4)
\end{gathered}
$$

in $\mathfrak{L}_{Q}(\mathbb{Z})$, where $\mathfrak{L}_{Q}(j)$ are the $j$-th filtered probability spaces, the free blocks of $\mathfrak{L}_{Q}(\mathbb{Z})$. In other words, this product operator $X$ in the free family $\mathcal{X}_{Q}$ is the free reduced word $U_{-1}^{2} U_{0} U_{-1} U_{4}^{3}$ in $\mathfrak{L}_{Q}(\mathbb{Z})$.

Therefore, indeed, the above lemma characterizes the full free-distributional data obtained from the free semicircular family $\mathcal{X}_{Q}$ of (6.5) in $\mathfrak{L}_{Q}(\mathbb{Z})$.

However, more precisely, we may refine the above results as follows. First, observe that if $\left(j_{1}\right.$, $\left.\ldots, j_{n}\right)$ is an alternating $n$-tuple in $\mathbb{Z}$, and if there exists a unique partition of the $n$-tuple $\left(j_{1}, \ldots, j_{n}\right)$ with $\mathrm{N}$-many noncrossing blocks

$$
\left(\left(j_{1}, \ldots, j_{n_{1}}\right),\left(j_{n_{1}+1}, \ldots, j_{n_{1}+n_{2}}\right), \ldots,\left(j_{n_{1}+\ldots+n_{N-1}+1}, \ldots, j_{n_{1}+\ldots+n_{N-1}+n_{N}}\right)\right),
$$


where

$$
\begin{gathered}
j_{1}=\ldots=j_{n_{1}} \\
j_{n_{1}+1}=\ldots=j_{n_{1}+n_{2}} \\
j_{n_{1}+\ldots+n_{N-1}+1}=\ldots=j_{n_{1}+\ldots+n_{N}}
\end{gathered}
$$

satisfying

$$
j_{1} \neq j_{n_{1}+1}, j_{n_{1}+1} \neq j_{n_{1}+n_{2}+1}, \ldots, j_{n_{1}+\ldots+n_{N-1}} \neq j_{n_{1}+\ldots+n_{N-1}+1}
$$

in $\mathbb{Z}$.

Then, we call such maximal partition of $\left(j_{1}, \ldots, j_{n}\right)$, the alternating partition.

For example, in the very above product operator $X$, one can induce the corresponding integer-sequence,

$$
(-1,-1,0,-1,4,4,4)
$$

with its alternating partition,

$$
((-1,-1),(0),(-1),(4,4,4)) \text {. }
$$

It is trivial that if an integer-sequence $\left(j_{1}, \ldots, j_{n}\right)$ is alternating in $\mathbb{Z}$, then its alternating partition is

$$
\left(\left(j_{1}\right),\left(j_{2}\right),\left(j_{3}\right), \ldots,\left(j_{n}\right)\right) .
$$

Now, let $W=\left(j_{1}, \ldots, j_{n}\right)$ be a finite integer sequence regarded as its unique alternating partition,

$$
W=\left(\left[j_{l_{1}}\right]_{1}, \ldots,\left[j_{l_{N}}\right]_{N}\right),
$$

where $\left[j_{l_{1}}\right], \ldots,\left[j_{l_{N}}\right]$ are the blocks of the alternating partition of $W$, with $N \leq n$ in $\mathbb{N}$, satisfying

$$
\left[j_{l_{s}}\right]_{s}=\left(j_{l_{s}}, j_{l_{s}}, \ldots, j_{l_{s}}\right) \text { in } W,
$$

for all $s=1, \ldots, N$, with

$$
\left[j_{1}\right]_{1}=\left[j_{1}\right]_{1} .
$$

We say that the cardinality $N$ of blocks in $W$ the (alternating-)partition size of $\boldsymbol{W}$. One can define the following quantities $\left|\left[j_{l_{s}}\right]\right|$ for a fixed size- $N$ alternating partition of the sequence $W$

$$
\left|\left[j_{l_{s}}\right]_{s}\right|=\text { the cardinality of }\left[j_{l_{s}}\right]_{s} \text { in } W
$$

for all $l=1, \ldots, N$. We call these quantities $\left|\left[j_{l_{s}}\right]_{s}\right|$ the block-sizes of $W$, for all $s=1, \ldots, N$.

For example, if the product operator $X$ is a free reduced word, $U_{-1}^{2} U_{0} U_{-1} U_{4}^{3}$ of $\mathfrak{L}_{Q}(\mathbb{Z})$ is as above inducing the size- 4 alternating partition of its integer-sequence,

$$
\left([-1]_{1},[0]_{2},[-1]_{3},[4]_{4}\right)=((-1,-1),(0),(-1),(4,4,4)),
$$

then

$$
\left|[-1]_{1}\right|=2,\left|[0]_{2}\right|=1=\left|[-1]_{3}\right|,
$$

and

$$
\left|[4]_{4}\right|=3 \text {. }
$$


We can realize that the block-sizes are identical to the powers of free-factors of $X$.

Example 1. Let $\mathcal{X}_{Q}=\left\{U_{j} \in \mathfrak{L}_{Q}(j)\right\}_{j \in \mathbb{Z}}$ be the free semicircular family (6.5) in the free filterization $\mathfrak{L}_{Q}(\mathbb{Z})$ of $Q$, and let

$$
X=U_{-1} U_{-1} U_{0} U_{-1} U_{4} U_{4} U_{4} \in \mathfrak{L}_{Q}(\mathbb{Z})
$$

be a product operator of $\mathfrak{L}_{Q}(\mathbb{Z})$ in $\mathcal{X}_{Q}$. Then, this operator $\boldsymbol{X}$ is identical to the free reduced word

$$
X=U_{-1}^{2} U_{0} U_{-1} U_{4}^{3} \text { in } \mathfrak{L}_{Q}(\mathbb{Z}),
$$

inducing the size-4 alternating partition of the corresponding integer-sequence,

$$
\left([-1]_{1},[0]_{2},[-1]_{3},[4]_{4}\right),
$$

with

$$
[-1]_{1}=(-1,-1),[0]_{2}=(0),[-1]_{3}=(-1),
$$

and

$$
[4]_{4}=(4,4,4),
$$

having the block-sizes

\section{$2,1,1$, and 3 ,}

respectively.

Based on the above new concepts we discussed, let's refine the computations (8.7) and (8.8).

Lemma 3. Let $U=\prod_{l=1}^{n} U_{j_{l}}$ be a product operator of $\mathfrak{L}_{Q}(\mathbb{Z})$ in the free semicircular family $\mathcal{X}_{Q}$ of (6.5), for $n \in \mathbb{N}$. Assume that $\boldsymbol{U}$ induces the size-N alternating-partition $\left(\left[j_{l_{1}}\right]_{1}, \ldots,\left[j_{l_{N}}\right]_{N}\right)$ of its integer-partition $\left(j_{1}, \ldots, j_{n}\right)$, with the block-sizes

$$
\left|\left[j_{l_{s}}\right]_{s}\right|=N_{s}, \text { for all } s=1, \ldots, N,
$$

with

$$
n=N_{1}+N_{2}+\ldots+N_{N} \text { in } \mathbb{N},
$$

for some $\boldsymbol{N} \leq \boldsymbol{n}$ in $\mathbb{N}$. Then, this product $\boldsymbol{U}$ is the free reduced word,

$$
U=\prod_{s=1}^{N} U_{j_{l_{s}}}^{N_{s}} \in \mathfrak{L}_{Q}(\mathbb{Z}),
$$

satisfying

$$
\tau(U)=\prod_{s=1}^{N}\left(\omega_{N_{s}} c_{\frac{N_{s}}{2}}\right)=\tau\left(U^{*}\right) .
$$

Proof. Let $U$ be given as above in the free filterization $\mathfrak{L}_{Q}(\mathbb{Z})$ of $Q$. Then, by the very above discussion, this product operator $U$ in $\mathcal{X}_{Q}$ is the free reduced word $\prod_{s=1}^{N} U_{j_{l_{s}}}^{N_{s}}$ in $\mathfrak{L}_{Q}(\mathbb{Z})$, where $N_{s}$ are the block-sizes of the size- $N$ alternating partition of $\left(j_{1}, \ldots, j_{n}\right)$ in $\mathbb{Z}$. Thus, the product $U$ is identified with the free reduced word of $(8.9)$ in $\mathfrak{L}_{Q}(\mathbb{Z})$.

By (8.9), (8.7) and (8.8), the free-moment computation (8.10) holds. 
By the above three lemmas, we obtain the following free-distributional data of the free sum $T$ in the sense of (8.1) in the $M$-free filterization $\mathfrak{L}_{Q}^{M}(\mathbb{Z})$.

Theorem 6. Let $T=\sum_{j \in S u p p(T)}\left(m_{j} \otimes u_{j}^{k_{j}}\right)$ be the free sum (8.1) in the $M$-free filterization $\mathfrak{L}_{Q}^{M}(\mathbb{Z})$. Then,

$$
\tau^{M}\left(T^{n}\right)=\sum_{\left(j_{1}, \ldots, j_{n}\right) \in S u p p(T)^{n}} \operatorname{tr}\left(\prod_{l=1}^{n} m_{j_{l}}\right) \tau\left(\prod_{l=1}^{n} u_{j_{l}}^{k_{j_{l}}}\right)
$$

for all $n \in \mathbb{N}$. In particular, for any fixed $\left(j_{1}, \ldots, j_{n}\right) \in \operatorname{Sup} p(T)^{n}$, one has the corresponding integer-sequence

$$
W_{j_{1}, \ldots, j_{n}}=(\underbrace{j_{1}, \ldots, j_{1}}_{k_{j_{1}}}, \underbrace{j_{2}, \ldots, j_{2}}_{k_{j_{2}}}, \ldots, \underbrace{j_{n}, \ldots, j_{n}}_{k_{j_{n}}}) .
$$

Then, the integer-sequence $\boldsymbol{W}_{j_{1}, \ldots, j_{n}}$ has its unique alternative-partition,

$$
W_{j_{1}, \ldots, j_{n}}=\left(\left[j_{l_{1}}\right]_{1}, \ldots,\left[j_{l_{N}}\right]_{N}\right),
$$

with

$$
\left|\left[j_{l_{s}}\right]_{s}\right|=N_{s}, \text { for all } s=1, \ldots, N,
$$

such that $\sum_{s=1}^{N} N_{s}=\sum_{l=1}^{n} k_{j_{l}}$ in $\mathbb{N}$, inducing the free reduced word,

$$
\prod_{l=1}^{n} u_{j_{l}}^{k_{j_{l}}}=\prod_{s=1}^{n} u_{j_{l_{s}}}^{N_{s}} \text { in } \mathfrak{L}_{Q}(\mathbb{Z}),
$$

satisfying

$$
\tau\left(\prod_{l=1}^{n} U_{j_{l}}^{k_{j_{l}}}\right)=\prod_{s=1}^{N} \omega_{N_{s}} c_{\frac{N_{s}}{2}}
$$

Proof. The formula (8.11) is proven by (8.5). The computation (8.12) is shown by (8.9) and (8.10).

The following corollary is a direct consequence of the above theorem.

Corollary 3. Let $\mathbf{T}$ be the free sum (8.1) in the $\boldsymbol{M}$-free filterization $\mathfrak{L}_{Q}^{M}(\mathbb{Z})$. Then,

$$
\tau^{M}\left(\left(T^{*}\right)^{n}\right)=\sum_{\left(j_{1}, \ldots, j_{n}\right) \in \operatorname{Supp}(T)^{n}} \operatorname{tr}\left(\prod_{l=1}^{n} m_{j_{l}}^{*}\right) \tau\left(\prod_{l=1}^{n} u_{j_{l}}^{k_{j_{l}}}\right)
$$

where $\tau\left(\prod_{l=1}^{n} U_{j_{l}}^{k_{j_{l}}}\right)$ satisfy (8.12), for all $\left(j_{1}, \ldots, j_{n}\right) \in \operatorname{Supp}(T)^{n}$, for all $n \in \mathbb{N}$.

Proof. Since $T=\sum_{j \in S u p p(T)}\left(m_{j} \otimes U_{j}^{k_{j}}\right)$ is a free sum in $\mathfrak{L}_{Q}^{M}(\mathbb{Z})$, one can get that

$$
T^{*}=\sum_{j \in \operatorname{Supp}(T)}\left(m_{j}^{*} \otimes U_{j}^{k_{j}}\right) \text { in } \mathfrak{L}_{Q}^{M}(\mathbb{Z}),
$$

by the self-adjointness of the semicircular elements $U_{j}$ in the free filterization $\mathfrak{L}_{Q}(\mathbb{Z})$ (under A 5.1), for all $j \in \mathbb{Z}$.

Therefore, similar to (8.11) and (8.12), the formula (8.13) holds.

The following result is immediately obtained by (8.12) and (8.13). 
Corollary 4. Let $T$ be the free sum (8.1) in $\mathfrak{L}_{O}^{M}(\mathbb{Z})$. Assume that $\boldsymbol{m}_{j} \in(\boldsymbol{M}, \boldsymbol{t r})$ are self-adjoint in $\boldsymbol{M}$, for all $j \in \operatorname{Supp}(T)$ in $\mathbb{Z}$. Then, the free distribution of $T$ is characterized by the free-moment sequence, $\left(\tau^{M}\left(T^{n}\right)\right)_{n=1}^{\infty}$, whose entries are determined by (8.12).

Proof. Under hypothesis, the free sum $T$ is self-adjoint in $\mathfrak{L}_{Q}^{M}(\mathbb{Z})$ in the sense that $T^{*}=T$. Thus, by (8.12) and (8.13), this corollary is proven.

Now, we generalize the free-distributional data (8.12) and (8.13).

Let $\mathbb{Z}^{N}=\left\{\left(j_{k}\right)_{k=1}^{N}: j_{k} \in \mathbb{Z}\right\}$, for all $N \in \mathbb{N}$. Define a subset $A l t\left(\mathbb{Z}^{N}\right)$ of $\mathbb{Z}^{N}$ by

$$
\operatorname{Alt}\left(\mathbb{Z}^{N}\right) \stackrel{\text { def }}{=}\left\{\left(j_{k}\right)_{k=1}^{N} \in \mathbb{Z}^{N} \mid\left(j_{k}\right)_{k=1}^{N} \text { is alternating }\right\},
$$

for all $N \in \mathbb{N}$.

For an arbitrarily fixed $N$-tuple $W=\left(j_{1}, \ldots, j_{N}\right)$ in $\mathbb{Z}^{N}$, let $X$ be an ordered $N$-tuple

$$
X=\left(m_{j_{1}}, \ldots, m_{j_{N}}\right),
$$

whose entries are from $(M, t r)$, i.e., $m_{j_{k}} \in(M, t r)$, for all $k=1, \ldots, N$.

In addition, for the $N$-tuple $W$, let

$$
\eta=\left(n_{j_{1}}, \ldots, n_{j_{N}}\right)
$$

be an $N$-tuple of natural numbers, $n_{j_{k}} \in \mathbb{N}$, for all $k=1, \ldots, N_{k}$, where $j_{1}, \ldots, j_{N}$ are the entries of $W$.

For such $N$-tuples $W, X$ and $\eta$, define an operator $T_{W}^{X, \eta}$ by

$$
T_{W}^{X, \eta}=\prod_{k=1}^{N}\left(m_{k} \otimes U_{j_{k}}^{n_{k}}\right)=\prod_{j \in W}\left(m_{j} \otimes U_{j}^{n_{j}}\right),
$$

in the $M$-free filterization $\mathfrak{L}_{Q}^{M}(\mathbb{Z})$, for all $k=1, \ldots, n$.

Now, let a fixed $N$-tuple $W$ is taken from $\operatorname{Alt}\left(\mathbb{Z}^{N}\right)$ of $(8.14)$, and let

$$
T_{W}^{X, \eta}=\prod_{j \in W}\left(m_{j} \otimes U_{j}^{n_{j}}\right),
$$

be in the sense of (8.15), contained in the Banach $*$-subalgebra

$$
\bigotimes_{j \in W} \mathfrak{L}_{Q}^{M}(j) \text { of } \mathfrak{L}_{Q}^{M}(\mathbb{Z})
$$

(again, see $[2,3,9])$.

Remark 4. If $\boldsymbol{W}$ is not an alternating $N$-tuple in $\mathbb{Z}^{N}$, for some $N \in \mathbb{N}$, equivalently, if

$$
W \in \mathbb{Z}^{N} \backslash \operatorname{Alt}\left(\mathbb{Z}^{N}\right),
$$

then one can decide the maximal partition of $\boldsymbol{W}$, whose blocks consist only of same integers. For instance, if

$$
W=(-1,-1,2,3,3,-1) \in \mathbb{Z}^{6} \backslash W \notin A l t\left(\mathbb{Z}^{6}\right) ，
$$

then one has the partitioned sequence,

$$
W_{o}=((-1,-1),(2),(3,3),(-1)),
$$

and give reduction on (8.8) for each block of $\boldsymbol{W}_{\text {o }}$. i.e., 


$$
\begin{aligned}
& T_{W}^{X, \eta \eta} \stackrel{\text { equi }}{=}\left(m_{-1} \otimes U_{-1}^{n_{-1}}\right)\left(m_{-1}^{\prime} \otimes U_{-1}^{n_{-1}^{\prime}}\right) \\
& \otimes\left(m_{2} \otimes U_{2}^{n_{2}}\right) \otimes\left(m_{3} \otimes U_{3}^{n_{3}}\right)\left(m_{3}^{\prime} \otimes u_{3}^{n_{3}^{\prime}}\right) \\
& \otimes\left(m_{-1}^{\prime \prime} \otimes U_{-1}^{n_{-1}^{\prime \prime}}\right),
\end{aligned}
$$

and, hence,

$$
\begin{aligned}
T_{W}^{X, \eta \eta} \stackrel{\text { equi }}{=}\left(m_{-1} m_{-1}^{\prime} \otimes U_{-1}^{n_{-1}+n_{-1}^{\prime}}\right) \otimes\left(m_{2} \otimes U_{2}^{n_{2}}\right) \\
\otimes\left(m_{3} m_{3}^{\prime} \otimes U_{3}^{n_{3}+n_{3}^{\prime}}\right) \otimes\left(m_{-1}^{\prime \prime} \otimes U_{-1}^{n_{-1}^{\prime \prime}}\right)
\end{aligned}
$$

in the free block

$$
\mathfrak{L}_{Q}^{M}(-1) \otimes_{\mathbb{C}} \mathfrak{L}_{Q}^{M}(2) \otimes_{\mathbb{C}} \mathfrak{L}_{Q}^{M}(3) \otimes_{\mathbb{C}} \mathfrak{L}_{Q}^{M}(-1),
$$

in $\mathfrak{L}_{Q}^{M}(\mathbb{Z})$, which is identified with

$$
\begin{aligned}
T_{W}^{X, \eta}=\left(m_{-1} m_{-1}^{\prime} \otimes U_{-1}^{n_{-1}+n_{-1}^{\prime}}\right)\left(m_{2} \otimes U_{2}^{n_{2}}\right) \\
\cdot\left(m_{3} m_{3}^{\prime} \otimes U_{3}^{n_{3}+n_{3}^{\prime}}\right)\left(m_{-1}^{\prime \prime} \otimes U_{-1}^{n_{-1}^{\prime \prime}}\right)
\end{aligned}
$$

in $\mathfrak{L}_{Q}^{M}(\mathbb{Z})$.

As we considered above, if $\boldsymbol{W} \in \mathbb{Z}^{N}$, then there exists a unique $\boldsymbol{W}^{\prime} \in \operatorname{Alt}\left(\mathbb{Z}^{N}\right)$, such that

$$
T_{W}^{X, \eta}=T_{W^{\prime}}^{X^{\prime}, \eta^{\prime}} \in \mathfrak{L}_{Q}^{M}(\mathbb{Z}),
$$

as a free reduced word.

It shows that, without loss of generality, one can reduce his interests in alternating sequences (instead of all sequences in $\mathbb{Z}^{N}$ ) in $\operatorname{Alt}\left(\mathbb{Z}^{N}\right)$, for $N \in \mathbb{N}$.

Consider free distributions of free random variables $T_{W}^{X, \eta}$ of (8.16), for $W \in \operatorname{Alt}\left(\mathbb{Z}^{N}\right)$.

Theorem 7. Let $\boldsymbol{W} \in \operatorname{Alt}\left(\mathbb{Z}^{N}\right)$, for some $N \in \mathbb{N}$, and let $T_{W}^{X, \eta}$ be the operator (8.16) in the $M$-free filterization $\mathfrak{L}_{Q}^{M}(\mathbb{Z})$. Then,

$$
\tau^{M}\left(T_{W}^{X, \eta}\right)=\prod_{j \in W} \omega_{n_{j}} c_{\frac{n_{j}}{2}} \operatorname{tr}\left(m_{j}\right)
$$

and

$$
\tau^{M}\left(\left(T_{W}^{X, \eta}\right)^{*}\right)=\prod_{j \in W}\left(\omega_{n_{j}} c_{\frac{n_{j}}{2}} \operatorname{tr}\left(m_{j}^{*}\right)\right)
$$

Moreover, if the fixed alternating $N$-tuple $W=\left(j_{1}, \ldots, j_{N}\right)$ satisfies

$$
j_{1} \neq j_{N} \text { in } \mathbb{Z}
$$

then

$$
\tau^{M}\left(\left(T_{W}^{X, \eta}\right)^{n}\right)=\prod_{j \in W}\left(\omega_{n_{j}} c_{\frac{n_{j}}{2}} \operatorname{tr}\left(m_{j}\right)\right)^{n}
$$

and 


$$
\tau^{M}\left(\left(\left(T_{W}^{X, \eta}\right)^{*}\right)^{n}\right)=\prod_{j \in W}\left(\omega_{n_{j}} c{\frac{n_{j}}{2}}_{t} \operatorname{tr}\left(m_{j}^{*}\right)\right)^{n}
$$

for all $n \in \mathbb{N}$.

Proof. By the alternating-ness of $W$, the operator $T_{W}^{X,{ }^{\prime \prime} \eta}$ forms a free reduced word in $\mathfrak{L}_{Q}^{M}(\mathbb{Z})$, by $(7.6)$. Therefore, one can get that

$$
\begin{aligned}
\tau^{M}\left(T_{W}^{X, \eta}\right) & =\prod_{j \in W} \tau_{j}^{M}\left(m_{j} \otimes u_{j}^{n_{j}}\right) \\
& =\prod_{j \in W}\left(\operatorname{tr}\left(m_{j}\right)\right)\left(\tau_{j}\left(u_{j}^{n_{j}}\right)\right) \\
& =\prod_{j \in W} \omega_{n_{j}} c_{\frac{n_{j}}{2}} \operatorname{tr}\left(m_{j}\right)
\end{aligned}
$$

by the semicircularity of $\boldsymbol{U}_{j}{ }^{\prime}$ s in $\mathfrak{L}_{Q}(\mathbb{Z})$. Similarly,

$$
\begin{aligned}
\tau^{M}\left(\left(T_{W}^{X, \eta}\right)^{*}\right) & =\prod_{j \in W} \tau_{j}^{M}\left(m_{j}^{*} \otimes U_{j}^{n_{j}}\right) \\
& =\prod_{j \in W} \omega_{n_{j}} c_{\frac{n_{j}}{2}} t r\left(m_{j}^{*}\right) .
\end{aligned}
$$

Thus, the free-distributional data (8.17) are obtained.

Assume now that a fixed alternating $N$-tuple $W=\left(j_{1}, \ldots, j_{N}\right)$ satisfies

$$
j_{1} \neq j_{N} \text { in } \mathbb{Z} \text {. }
$$

Under this additional condition, one can realize that the $n N$-tuples

$$
W^{n}=(\underbrace{W, W, \ldots, W}_{n \text {-times }}) \in \mathbb{Z}^{n N}
$$

satisfy

$$
W^{n} \in \operatorname{Alt}\left(\mathbb{Z}^{n N}\right), \text { for all } n \in \mathbb{N} \text {, }
$$

i.e., $W^{n}$ are alternating in $\mathbb{Z}$, for all $n \in \mathbb{N}$. It guarantees that the operators $\left(T_{W}^{X, \eta}\right)^{n}$ form free reduced words in $\mathfrak{L}_{Q}^{M}(\mathbb{Z})$, for all $n \in \mathbb{N}$. Therefore,

$$
\begin{aligned}
\tau^{M}\left(\left(T_{W}^{X, \eta}\right)^{n}\right) & =\tau^{M}(\underbrace{T_{W}^{X, \eta} \cdots T_{W}^{X, \eta}}_{n \text {-times }}) \\
& =\left(\tau^{M}\left(T_{W}^{X, \eta}\right)\right)^{n}=\left(\prod_{j \in W} \omega_{n_{j}} c c_{\frac{n_{j}}{2}} t r\left(m_{j}\right)\right)^{n},
\end{aligned}
$$

and, similarly,

$$
\tau^{M}\left(\left(\left(T_{W}^{X, \eta}\right)^{*}\right)^{n}\right)=\left(\prod_{j \in W} \omega_{n_{j}} c n_{\frac{n_{j}}{2}} t r\left(m_{j}^{*}\right)\right)^{n}
$$

for all $n \in \mathbb{N}$, by (8.17).

Therefore, the free-probabilistic information (8.18) is obtained. 
As we have seen above, our main results of Section 8, the free-distributional data induced by the free semicircular family $\mathcal{X}$, are affected by the freeness (6.3) on the free filterization $\mathfrak{L}_{Q}(\mathbb{Z})$ of $Q$ in the $M$-free filterization $\mathfrak{L}_{Q}^{M}(\mathbb{Z})$. In Section 9, let us consider freeness conditions and corresponding free-distributional information on $\mathfrak{L}_{Q}^{M}(\mathbb{Z})$ affected by the freeness on $(M, t r)$.

\section{Certain Freeness Conditions on $\left(\mathfrak{L}_{Q}^{M}(\mathbb{Z}), \tau^{M}\right)$}

In this section, we consider freeness conditions on our $\boldsymbol{M}$-free filterization

$$
\mathfrak{L}_{Q}^{M}(\mathbb{Z})=\left(\mathfrak{L}_{Q}^{M}(\mathbb{Z}), \tau^{M}\right),
$$

affected by the freeness on a fixed unital tracial $W^{*}$-probability space $(M, t r)$.

Since $\mathfrak{L}_{Q}(\mathbb{Z})$ is defined to be the free product of $j$-th filtered probability spaces $\left\{\mathfrak{L}_{Q}(j)\right\}_{j \in \mathbb{Z}}$, the freeness (6.3) on the free filterization $\mathfrak{L}_{Q}(\mathbb{Z})$ of $Q$ affects the free-distributional information on $\mathfrak{L}_{Q}^{M}(\mathbb{Z})$ canonically (see Section 8; e.g., (8.12), (8.13), (8.17) and (8.18)), and it affects the freeness on $\mathfrak{L}_{Q}^{M}(\mathbb{Z})$ (see Section 7; e.g., (7.11), (7.12), (7.15) and (7.16)).Therefore, it is natural to ask how the freeness on the other tensor-factor $(M, t r)$ affects the freeness on the $M$-free filterization $\mathfrak{L}_{Q}^{M}(\mathbb{Z})$.

Assume that a fixed $W^{*}$-probability space $(M, t r)$ satisfies

$$
\begin{aligned}
(M, t r) & =\left(M_{1} \star M_{2}, t r_{1} \star t r_{2}\right) \\
& =\left(M_{1}, t r_{1}\right) \star\left(M_{2}, t r_{2}\right) .
\end{aligned}
$$

Then, the free blocks $\mathfrak{L}_{Q}^{M}(j)$ of the $M$-free filterization $\mathfrak{L}_{Q}^{M}(\mathbb{Z})$ satisfies that

$$
\mathfrak{L}_{Q}^{M}(j) \stackrel{\text { def }}{=} M \otimes_{\mathbb{C}} \mathfrak{L}_{Q}(j),
$$

where $\mathfrak{L}_{Q}(j)$ are the $j$-th filtered probability spaces, the free blocks of the free filterization $\mathfrak{L}_{Q}(\mathbb{Z})$ of $Q$

by $(9.1)$

$$
=\left(M_{1} \star M_{2}\right) \otimes_{\mathbb{C}} \mathfrak{L}_{Q}(j)
$$

$$
=\left(M_{1} \star M_{2}\right) \otimes_{\mathbb{C}}\left(\mathfrak{L} \otimes_{\mathbb{C}} Q\right)
$$

because, as a Banach $*$-algebra, $\mathfrak{L}_{Q}(j)=\mathfrak{L}_{Q}$, the projection radial algebra (for each $j \in \mathbb{Z}$ ), where $\mathfrak{L}$ is the radial algebra (3.12), by (3.14)

by $(3.8)$

$$
\stackrel{* \text {-iso }}{=}\left(M_{1} \star M_{2}\right) \otimes_{\mathbb{C}}\left(\mathfrak{L} \otimes_{\mathbb{C}} \mathbb{C}^{\oplus|\mathbb{Z}|}\right)
$$

$$
\begin{aligned}
& \stackrel{* \text {-iso }}{=}\left(M_{1} \star M_{2}\right) \otimes_{\mathbb{C}}\left(\mathfrak{L}^{\oplus|\mathbb{Z}|}\right) \\
& \stackrel{* \text {-iso }}{=}\left(\left(M_{1} \star M_{2}\right) \otimes_{\mathbb{C}} \mathfrak{L}\right)^{\oplus|\mathbb{Z}|} \\
& \left.\stackrel{\text { def }}{=}\left(\left(M_{1} \star M_{2}\right) \otimes_{\mathbb{C}} \overline{\mathbb{C}[\{l\}]}\right)^{\|\cdot\|}\right)^{\oplus|\mathbb{Z}|}
\end{aligned}
$$

by (3.12)

$$
\stackrel{* \text {-iso }}{=}\left(\overline{\left(M_{1} \star M_{2}\right)[\{l\}]}\right)^{\oplus|\mathbb{Z}|} \text {, }
$$

where $M[\{l\}]$ means the polynomial-algebra in $l$ with $M$-coefficients, and where $\bar{Y}$ means the norm-topology-closure under the product topology for the $W^{*}$-topology for $M_{1} \star M_{2}$ (or that for M) and the Banach-topology for $\overline{\mathbb{C}}[\{l\}]^{\|\cdot\|}$ in the sense of (3.12) 


$$
\begin{aligned}
& \stackrel{* \text {-iso }}{=}\left(\overline{M_{1}[\{l\}] \star M_{2}[\{l\}]}\right)^{\oplus|\mathbb{Z}|} \\
& =\left(\overline{M_{1}[\{l\}]} \star \overline{M_{2}[\{l\}]}\right)^{\oplus|\mathbb{Z}|} \\
& \stackrel{* \text {-iso }}{=}\left(\left(M_{1} \otimes_{\mathbb{C}} \mathfrak{L}\right) \star\left(M_{\mathbf{2}} \otimes_{\mathbb{C}} \mathfrak{L}\right)\right)^{\oplus|\mathbb{Z}|} \\
& \stackrel{* \text {-iso }}{=}\left(M_{1} \otimes_{\mathbb{C}} \mathfrak{L}\right)^{\oplus|\mathbb{Z}|} \star\left(M_{2} \otimes_{\mathbb{C}} \mathfrak{L}\right)^{\oplus|\mathbb{Z}|} \\
& \stackrel{* \text {-iso }}{=}\left(M_{1} \otimes_{\mathbb{C}} \mathfrak{L}^{\oplus|\mathbb{Z}|}\right) \star\left(M_{2} \otimes_{\mathbb{C}} \mathfrak{L}^{\oplus|\mathbb{Z}|}\right) \\
& \stackrel{\text { *-iso }}{=}\left(M_{1} \otimes_{\mathbb{C}} \mathfrak{L}_{Q}\right) \star\left(M_{\mathbf{2}} \otimes_{\mathbb{C}} \mathfrak{L}_{Q}\right) \\
& =\left(M_{\mathbf{1}} \otimes_{\mathbb{C}} \mathfrak{L}_{Q}(j)\right) \star\left(\boldsymbol{M}_{\mathbf{2}} \otimes_{\mathbb{C}} \mathfrak{L}_{Q}(j)\right) \\
& =\mathfrak{L}_{Q}^{M_{1}}(j) \star \mathfrak{L}_{Q}^{M_{2}}(j),
\end{aligned}
$$

where $\mathfrak{L}_{Q}^{M_{l}}(j)$ are in the sense of (7.3), equipped with their linear functionals

$$
\tau_{j}^{M_{l}}=\operatorname{tr}_{l} \otimes \tau_{j}
$$

in the sense of (7.4), for all $l=1,2$, for all $j \in \mathbb{Z}$.

By (9.2) and (9.3), we obtain the following structure theorems.

Theorem 8. Let $j \in \mathbb{Z}$ be arbitrarily fixed, and let $\mathfrak{L}_{Q}^{M}(j)$ be the corresponding free block of the $M$-free filterization $\mathfrak{L}_{Q}^{M}(\mathbb{Z})$ of $Q$. Assume that a fixed unital tracial $W^{*}$-probability space $(M, t r)$ satisfies the freeness (9.1). Then,

$$
\mathfrak{L}_{Q}^{M}(j)=\mathfrak{L}_{Q}^{M_{1} \star M_{2}}(j) \stackrel{*-i s o}{=} \mathfrak{L}_{Q}^{M_{1}}(j) \star \mathfrak{L}_{Q}^{M_{2}}(j),
$$

where

$$
\mathfrak{L}_{Q}^{M_{l}}(j)=\left(\mathfrak{L}_{Q}^{M_{l}}, \tau_{j}^{M_{l}}\right)=\left(M \otimes_{\mathbb{C}} \mathfrak{L}_{Q}, t r_{l} \otimes \tau_{j}\right),
$$

for all $l=1,2$.

Proof. The structure theorem (9.4) is proven by (9.2), where $\mathfrak{L}_{Q}^{M_{l}}(j)$ are the Banach $*$-algebras $\mathfrak{L}_{Q}^{M_{l}}$ in the sense of (7.3) equipped with their linear functionals $\tau_{j}^{M_{l}}$ of (9.3) as in (7.4).

By (9.4), one can get the following corollary immediately.

Corollary 5. Suppose a given $\boldsymbol{W}^{*}$-probability space $(\boldsymbol{M}, \boldsymbol{t r})$ is the free product $\mathbf{W}^{*}$-probability space,

$$
(M, t r)=\underset{s \in \Lambda}{\star}\left(M_{s}, t r_{s}\right),
$$

for an countable (finite or infinite) index set $\Lambda$ (under suitable product topology). Then, the free blocks $\mathfrak{L}_{Q}^{M}(j)$ of the $M$-free filterization $\mathfrak{L}_{Q}^{M}(\mathbb{Z})$ of $Q$ satisfy that

$$
\mathfrak{L}_{Q}^{M}(j) \stackrel{*-i s o}{=} \underset{s \in \Lambda}{\star} \mathfrak{L}_{Q}^{M_{s}}(j), \text { for all } j \in \mathbb{Z},
$$


where

$$
\mathfrak{L}_{Q}^{M_{s}}(j)=\left(\mathfrak{L}_{Q}^{M_{s}}=M_{s} \otimes_{\mathbb{C}} \mathfrak{L}_{Q}(j), \tau_{j}^{M_{s}}=\operatorname{tr}_{s} \otimes \tau_{j}\right),
$$

for all $s \in \Lambda$.

Proof. The structure theorem (9.6) is obtained by the induction on (9.4), where $(M, t r)$ satisfies the freeness (9.5).

By (9.4) and (9.6), one can get the following structure theorem for our $M$-free filterization $\mathfrak{L}_{Q}^{M}(\mathbb{Z})$ in terms of the freeness on $(M, t r)$.

Corollary 6. Let $(M, t r)=\underset{s \in \Lambda}{\star}\left(M_{s}, t r_{s}\right)$, and let $\mathfrak{L}_{Q}^{M}(\mathbb{Z})$ be the $M$-free filterization of $Q$, where $\Lambda$ is a (finite, or infinite) countable index set. Then,



Proof. Observe that

$$
\begin{aligned}
& \mathfrak{L}_{Q}^{M}(\mathbb{Z}) \stackrel{\text { def }}{=} \underset{j \in \mathbb{Z}}{\star} \mathfrak{L}_{Q}^{M}(j)=\underset{j \in \mathbb{Z}}{\star}\left(\mathfrak{L}_{Q}^{\stackrel{\star}{\mathfrak{s}^{\star \Lambda \Lambda}} M^{M_{s}}}(j)\right)
\end{aligned}
$$

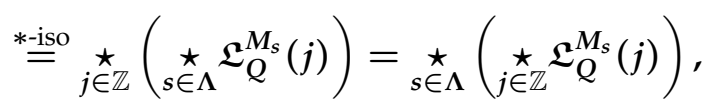

by (9.6). Thus, the $*$-isomorphic relation (9.7) holds.

The above structure theorem (9.7) characterizes the freeness on the $M$-free filterization $\mathfrak{L}_{Q}^{M}(\mathbb{Z})$ by the freeness on $(M, t r)$. In fact, it shows how the freeness both on $(M, t r)$ and on the free filterization $\mathfrak{L}_{Q}(\mathbb{Z})$ of $Q$ affect the free structure of $\mathfrak{L}_{Q}^{M}(\mathbb{Z})$.

In addition, the structure theorem (9.4), and its generalization (9.6) shows the following results as well.

Theorem 9. Let $\boldsymbol{y}_{1}, \boldsymbol{y}_{2}$ be free random variables in a fixed $\boldsymbol{W}^{*}$-probability space $(\boldsymbol{M}, \boldsymbol{t r})$, and suppose they are free in $(M, t r)$. Then, the corresponding operators

$$
y_{1, j}^{n_{1}}=y_{1} \otimes u_{j}^{n_{1}} \text { and } y_{2, j}^{n_{2}}=y_{2} \otimes u_{j}^{n_{2}}
$$

are free in the $\boldsymbol{M}$-free filterization $\mathfrak{L}_{Q}^{M}(\mathbb{Z})$ of $Q$, for any arbitrarily fixed $j \in \mathbb{Z}$, for all $\boldsymbol{n}_{\mathbf{1}}, \boldsymbol{n}_{\mathbf{2}} \in \mathbb{N}$. i.e., for all $j$ $\in \mathbb{Z}$,

$$
y_{1} \text { and } y_{2} \text { are free in }(M, t r) \Rightarrow y_{1, j}^{n_{1}} \text { and } y_{2, j}^{n_{2}} \text { are free in } \mathfrak{L}_{Q}^{M}(\mathbb{Z})
$$

Proof. Now, let $y_{l, j}^{n_{l}} \in \mathfrak{L}_{Q}^{M}(\mathbb{Z})$ be in the sense of (9.8), for all $l=1,2$, where the tensor-factors $y_{l}$ of them are free in $(M, t r)$. Now, construct $W^{*}$-subalgebras $M_{y_{l}}$ of $M$ by

$$
M_{y_{l}}=W^{*}\left(\left\{y_{l}, y_{l}^{*}\right\}\right), \text { for all } l=1,2,
$$

and consider the restricted linear functionals

$$
t r_{l}=\left.\operatorname{tr}\right|_{M_{y_{l}}}, \text { for all } l=1,2 .
$$

Then, it is not difficult to check that the $W^{*}$-subalgebra 


$$
M_{y_{1}, y_{2}}=W^{*}\left(\left\{y_{1}, y_{1}^{*}, y_{2}, y_{2}^{*}\right\}\right)
$$

satisfies

$$
M_{y_{1}, y_{2}}=M_{y_{1}} \star M_{y_{2}} \text { in }(M, t r)
$$

with respect to the linear functional

$$
\operatorname{tr} r_{1,2}=\left.\operatorname{tr}\right|_{M_{y_{1}, y_{2}}} \text { on } M_{y_{1}, y_{2}}
$$

satisfying

$$
\operatorname{tr}_{1,2}=\operatorname{tr}_{1} \star t r_{2} \text { on } M_{y_{1}, y_{2}} \text {. }
$$

Now, we consider $M_{y_{1}, y_{2}}$-free filterization $\mathfrak{L}_{Q}^{M_{y_{1}, y_{2}}}(\mathbb{Z})$, as a Banach $*$-subalgebra of $\mathfrak{L}_{Q}^{M}(\mathbb{Z})$.

Then, by (9.4) and (9.6), we obtain that the free blocks $\mathfrak{L}_{Q}^{M_{y_{1}, y_{2}}}(j)$ of $\mathfrak{L}_{Q}^{M_{y_{1}, y_{2}}}(\mathbb{Z})$ satisfy

$$
\mathfrak{L}_{Q}^{M_{y_{1}, y_{2}}}(j)=\mathfrak{L}_{Q}^{M_{y_{1}} \star M_{y_{2}}}(j)=\mathfrak{L}_{Q}^{M_{y_{1}}}(j) \star \mathfrak{L}_{Q}^{M_{y_{2}}}(j),
$$

in $\mathfrak{L}_{Q}^{M_{y_{1}, y_{2}}}(\mathbb{Z})$ (inside $\mathfrak{L}_{Q}^{M}(\mathbb{Z})$ ), for all $j \in \mathbb{Z}$.

Note that

$$
y_{l, j^{\prime}}^{n_{l}}\left(y_{l, j}^{n_{l}}\right)^{*} \in \mathfrak{L}_{Q}^{M_{y_{l}}}(j) \subset \mathfrak{L}_{Q}^{M_{y_{1}, y_{2}}}(j) \subset \mathfrak{L}_{Q}^{M}(j),
$$

in $\mathfrak{L}_{Q}^{M}(\mathbb{Z})$, for all $l=1,2$.

By (9.10) and (9.11), the subsets

$$
\left\{y_{1, j^{\prime}}^{n_{1}},\left(y_{1, j}^{n_{1}}\right)^{*}\right\} \text { and }\left\{y_{2, j^{\prime}}^{n_{2}}\left(y_{2, j}^{n_{2}}\right)^{*}\right\}
$$

are contained in the distinct free blocks $\mathfrak{L}_{Q}^{M_{y_{1}}}(j)$, respectively $\mathfrak{L}_{Q}^{M_{y_{2}}}(j)$, in the free block $\mathfrak{L}_{Q}^{M}(j)$ of $\mathfrak{L}_{Q}^{M}(\mathbb{Z})$. It shows that these two subsets are free in $\mathfrak{L}_{Q}^{M}(\mathbb{Z})$ (e.g., [9-11]), equivalently, the operators $y_{1, j}^{n_{1}}$ and $y_{2, j}^{n_{2}}$ of $(9.8)$ are free in $\mathfrak{L}_{Q}^{M}(\mathbb{Z})$.

Therefore, the statement (9.9) holds.

The true statement (9.9) shows that the freeness conditions on $(M, t r)$ implies the freeness on free blocks of the $M$-free filterization $\mathfrak{L}_{Q}^{M}(\mathbb{Z})$ of $Q$. Note that the freeness condition (9.9) is slightly different from the structure theorem (9.7). The above statement (9.9) shows that the "embedded" free structures in $(M, t r)$ affects the freeness on $\mathfrak{L}_{Q}^{M}(\mathbb{Z})$; meanwhile, the structure theorem (9.7) says the freeness on $(M, t r)$ affects the freeness on $\mathfrak{L}_{O}^{M}(\mathbb{Z})$.

By (9.7), or by (9.9), we obtain the following free-distributional data.

Theorem 10. Let $y_{1}, y_{2}$ be free random variables in a given $W^{*}$-probability space $(\boldsymbol{M}, \boldsymbol{t r})$, and suppose that they are free in $(\boldsymbol{M}, \boldsymbol{t r})$. Let $\boldsymbol{y}_{l, j}=\boldsymbol{y}_{l, j}^{1}$ is in the sense of $(9.8)$ in the $\boldsymbol{M}$-free filterization $\mathfrak{L}_{Q}^{M}(\mathbb{Z})$ of $Q$, for all $l=1,2$. Assume that

$$
\left(i_{1}, \ldots, i_{n}\right) \in\{1,2\}^{n}, \text { for } n \in \mathbb{N}
$$

is an alternating $\boldsymbol{n}$-tuple in $\{\mathbf{1}, \mathbf{2}\}$. Let

$$
T=\prod_{s=1}^{n} y_{l_{i_{s}} j} \in \mathfrak{L}_{Q}^{M}(\mathbb{Z}) .
$$

Then,

$$
\tau^{M}(T)=\omega_{n} c_{\frac{n}{2}}\left(\prod_{s=1}^{n} \operatorname{tr}\left(y_{l_{s}}\right)\right)
$$


Moreover, if $\boldsymbol{i}_{\mathbf{1}} \neq \boldsymbol{i}_{n}$ in $\{\mathbf{1}, \mathbf{2}\}$, where $\boldsymbol{i}_{\mathbf{1}}$ and $\boldsymbol{i}_{\boldsymbol{n}}$ are the entries of the $\boldsymbol{n}$-tuple of (9.12), then

$$
\tau^{M}\left(T^{N}\right)=\left(\omega_{n} c \frac{n}{2}\right)^{N} \prod_{s=1}^{n}\left(\operatorname{tr}\left(y_{l_{i s}}\right)\right)^{N}, \text { for all } N \in \mathbb{N} .
$$

Proof. Suppose $T$ be in the sense of (9.13) in $\mathfrak{L}_{Q}^{M}(\mathbb{Z})$ under the alternating condition (9.12). Then, this operator $T$ is contained in the free block $\mathfrak{L}_{Q}^{M}(j)$ of $\mathfrak{L}_{Q}^{M}(\mathbb{Z})$. By regarding $T$ as an element in $\mathfrak{L}_{Q}^{M}(j)$, it is regarded as a free reduced word in $\mathfrak{L}_{Q}^{M}(j)$ by $(9.9)$ and (9.10) (e.g., [2,3,9]). Therefore,

$$
\begin{aligned}
\tau^{M}(T)=\tau_{j}^{M}\left(\prod_{s=1}^{n} y_{l_{i_{s}}, j}\right)=\tau_{j}^{M}\left(\prod_{s=1}^{n}\left(y_{l_{i_{s}}} \otimes U_{j}\right)\right) \\
=\tau_{j}^{M}\left(\left(\prod_{s=1}^{n} y_{l_{i_{s}}}\right) \otimes U_{j}^{n}\right)=\operatorname{tr}\left(\prod_{s=1}^{n} y_{l_{i_{s}}}\right)\left(\tau_{j}\left(U_{j}^{n}\right)\right) \\
=\left(\omega_{n} c \frac{n}{2}\right) \operatorname{tr}\left(\prod_{s=1}^{n} y_{l_{i_{s}}}\right) \\
=\left(\omega_{n} c_{\frac{n}{2}}\right)\left(\prod_{s=1}^{n} \operatorname{tr}\left(y_{l_{i_{s}}}\right)\right)
\end{aligned}
$$

by the alternating-ness of $\left(i_{1}, \ldots, i_{n}\right)$ in $\{\mathbf{1}, \mathbf{2}\}$. Therefore, the free-distributional data (9.14) holds.

Now, assume that $\left(i_{1}, \ldots, i_{n}\right)$ is not only alternating in $\{\mathbf{1}, \mathbf{2}\}$, but also

$$
i_{1} \neq i_{n} \text { in }\{1,2\} \text {. }
$$

Under this assumption, the operators $T^{N} \in \mathfrak{L}_{Q}^{M}(\mathbb{Z})$ are understood as free reduced words in the free block $\mathfrak{L}_{Q}^{M}(j)$, for all $N \in \mathbb{N}$. Therefore, one obtains that

$$
\tau^{M}\left(T^{N}\right)=\left(\tau^{M}(T)\right)^{N}, \text { for all } N \in \mathbb{N} .
$$

Therefore, the free-momental information (9.15) holds.

Remark 5. Free-distributional data (8.11), (8.12), (8.13), (8.17) and (8.18) shows how the freeness on the free filterization $\mathfrak{L}_{Q}(\mathbb{Z})$ of $Q$ affect the free-distributional data on the $\boldsymbol{M}$-free filterization $\mathfrak{L}_{Q}^{M}(\mathbb{Z})$. In addition, the free-distributional data (9.14) and (9.15) illustrate how the freeness on $(\boldsymbol{M}, \boldsymbol{t r})$ affects the free-distributional information on $\mathfrak{L}_{Q}^{M}(\mathbb{Z})$. By combining these results with (9.7), or with (9.9), one can characterize the free-probabilistic information of operators of $\mathfrak{L}_{Q}^{M}(\mathbb{Z})$, under freeness on $(M$, tr $)$, and that on $\mathfrak{L}_{Q}(\mathbb{Z})$.

\section{Application}

Let $(M, t r)$ be an arbitrarily fixed unital tracial $W^{*}$-probability space, and let $\mathfrak{L}_{Q}^{M}(\mathbb{Z})=\left(\mathfrak{L}_{Q}^{M}(\mathbb{Z}), \tau^{M}\right)$ be the $M$-free filterization of $Q$, where $Q$ is the $C^{*}$-subalgebra of a $C^{*}$-probability space $(A, \psi)$ generated by mutually-orthogonal $|\mathbb{Z}|$-many projections in $A$.

Now, we will apply our main results of Sections 7,8 and 9 to a special case, where a von Neumann algebra $M$ is given to be a free group factor, i.e.,

$$
M=L\left(F_{n}\right),
$$

generated by the free group $F_{n}$ of $n$-generators for $n \in \mathbb{N}_{\infty}^{>1}$, where

$$
\mathbb{N}_{\infty}^{>1} \stackrel{\text { def }}{=}(\mathbb{N} \backslash\{1\}) \cup\{\infty\}
$$


(e.g., [1,11]). For example, the free group factor $L\left(\boldsymbol{F}_{n}\right)$ is a group von Neumann algebra generated by $\boldsymbol{F}_{n}$, as a $\boldsymbol{W}^{*}$-subalgebra of the operator algebra $\boldsymbol{B}\left(\boldsymbol{l}^{2}\left(F_{n}\right)\right)$, where $\boldsymbol{l}^{2}\left(F_{n}\right)$ is the $\boldsymbol{l}^{2}$-Hilbert space generated by the group $F_{n}$, satisfying the following factor-ness.

A von Neumann algebra $\boldsymbol{M}$ (contained in the operator algebra $\boldsymbol{B}(\boldsymbol{H})$ of all operators on a Hilbert space $\boldsymbol{H}$ ) is a factor, if

$$
M \cap M^{\prime}=\mathbb{C} \cdot \mathbf{1}_{B(H)} \stackrel{\text { *-iso }}{=} \mathbb{C},
$$

where $\boldsymbol{M}^{\prime}$ is the commutant of $\boldsymbol{M}$ in $\boldsymbol{B}(\boldsymbol{H})$,

$$
M^{\prime} \stackrel{\text { def }}{=}\{T \in B(H): T m=m T, \forall m \in M\} .
$$

Recall that every von Neumann algebra $\boldsymbol{M}$ is decomposed by factors of different types. For more about von Neumann algebras and factors, see [11]. Note also that the free group factors $L\left(F_{n}\right)$ are indeed well-determined factors (e.g., [1] because $\boldsymbol{F}_{n}$ is an i.c.c. group), for all $n \in \mathbb{N}_{\infty}^{>1}$.

By construction, all elements $m$ of $L\left(F_{n}\right)$ are expressed by

$$
m=\sum_{g \in F_{n}} t_{g} g, \text { with } t_{g} \in \mathbb{C},
$$

in $L\left(F_{n}\right)$ (as finite sums or infinite sums under limit), with their adjoint,

$$
m^{*}=\sum_{g \in F_{n}} \overline{t_{g}} g^{*}=\sum_{g \in F_{n}} \overline{t_{g}} g^{-1},
$$

where $g^{*}$ is the adjoint of $g$ (as an operator in $L\left(F_{n}\right)$ ), and $g^{-1}$ is the group-inverse of $g$ (as a group-element of $\boldsymbol{F}_{\boldsymbol{n}}$ ).

The free group factors $L\left(F_{n}\right)$ are equipped with their canonical traces $t r_{n}$ on them, defined by

$$
t r_{n}\left(\sum_{g \in F_{n}} t_{g} g\right) \stackrel{d e f}{=} t_{e_{n}}
$$

where $e_{n}$ are the group-identities of $\boldsymbol{F}_{n}$, for all $n \in \mathbb{N}_{\infty}^{>1}$. i.e., if $m \in L\left(F_{n}\right)$, as a (possibly infinite) linear combination in $F_{n}$, then $\operatorname{tr}_{n}(m)$ is regarded as the process taking the coefficient $t_{e_{n}}$ of $m$, for the group identity $\boldsymbol{e}_{n}$ of $\boldsymbol{F}_{n}$.

Therefore, every free group factor $L\left(F_{n}\right)$ is automatically understood as a $W^{*}$-probability space $\left(L\left(F_{n}\right), t r_{n}\right)$, where $t r_{n}$ is the canonical trace (10.1) on $L\left(F_{n}\right)$, for all $n \in \mathbb{N}_{\infty}^{>1}$. From below, if we write $L\left(F_{n}\right)$, then it means either the free group factor, or the corresponding $W^{*}$-probability space $\left(L\left(F_{n}\right), t r_{n}\right)$.

It is not hard to check that $L\left(F_{n}\right)$ forms a unital tracial $W^{*}$-probability spaces, for all $n \in \mathbb{N}_{\infty}^{>1}$. Thus, under our settings, one can establish the corresponding $L\left(F_{n}\right)$-free filterization $\mathfrak{L}_{q}^{L\left(F_{n}\right)}(\mathbb{Z})$ of $Q$.

Notation Denote the $L\left(F_{n}\right)$-free filterizations $\mathfrak{L}_{Q}^{L\left(F_{n}\right)}(\mathbb{Z})$ simply by $\mathfrak{L}_{Q}(n, \mathbb{Z})$, for all $n \in \mathbb{N}_{\infty}^{>1}$.

It is well-known that, if $n \in \mathbb{N}_{\infty}^{>1}$, and if $n_{1}, n_{2} \in \mathbb{N}_{\infty}=\mathbb{N} \cup\{\infty\}$, such that

$$
n=n_{1}+n_{2} \text { in } \mathbb{N}_{\infty},
$$

then

$$
L\left(F_{n}\right) \stackrel{* \text {-iso }}{=} L\left(F_{n_{1}}\right) \star L\left(F_{n_{2}}\right)
$$

(e.g., see [1,3]), where $\stackrel{\text { "-iso” }}{=}$ means "being $W^{*}$-algebra-isomorphic".

More generally, if $n_{1}+n_{2}+\ldots+n_{k}=n$ in $\mathbb{N}_{\infty}^{>1}$, with $n_{1}, \ldots, n_{k} \in \mathbb{N}_{\infty}$, for $k \in \mathbb{N}$, then 


$$
L\left(F_{n}\right) \stackrel{* \text {-iso }}{=} \underset{l=1}{\star} L\left(F_{n_{l}}\right)
$$

by the induction on (10.2). For instance, by (10.3),

$$
L\left(F_{n}\right) \stackrel{* \text {-iso }}{=} \underbrace{L(\mathbb{Z}) \star L(\mathbb{Z}) \star \ldots \star L(\mathbb{Z})}_{n \text {-times }},
$$

for all $n \in \mathbb{N}_{\infty}^{>1}$, by regarding $\mathbb{Z}$ as the infinite cyclic abelian group $(\mathbb{Z},+)$ (up to group-isomorphisms). Radulescu showed in [8] that either the statement (10.4) or (10.5) holds true, where

$$
\begin{gathered}
L\left(F_{n_{1}}\right) \stackrel{* \text {-iso }}{=} L\left(F_{n_{2}}\right) \text {, for all } n_{1}, n_{2} \in \mathbb{N}_{\infty}^{>1}, \\
L\left(F_{n_{1}}\right) \stackrel{* \text {-iso }}{\neq} L\left(F_{n_{2}}\right), \text { whenever } n_{1} \neq n_{2} \text { in } \mathbb{N}_{\infty}^{>1} .
\end{gathered}
$$

Unfortunately, we do not know which one holds yet.

By (10.3) and (9.7), we obtain the following structure theorem of $\mathfrak{L}_{Q}(n, \mathbb{Z})$, for $n \in \mathbb{N}_{\infty}^{>1}$.

Corollary 7. Let $\mathfrak{L}_{Q}(n, \mathbb{Z})$ be the $L\left(F_{n}\right)$-free filterization of $Q$, for $n \in \mathbb{N}_{\infty}^{>1}$, and assume that

$$
\boldsymbol{n}=n_{1}+\ldots+n_{k} \text { in } \mathbb{N}_{\infty}, \text { for } n_{1}, \ldots, n_{k} \in \mathbb{N}_{\infty} .
$$

Then,

$$
\begin{aligned}
& \mathfrak{L}_{Q}(n, \mathbb{Z}) \stackrel{*-i s o}{=} \underset{l=1}{\star}\left(\underset{j \in \mathbb{Z}}{\star}\left(L\left(F_{n_{l}}\right) \otimes_{\mathbb{C}} \mathfrak{L}_{Q}(j)\right)\right) \\
& \stackrel{* \text {-iso }}{=} \underset{j \in \mathbb{Z}}{\star}\left(\underset{l=1}{\stackrel{n}{\star}}\left(L\left(F_{n_{l}}\right) \otimes \mathfrak{L}_{Q}(j)\right)\right),
\end{aligned}
$$

where $\mathfrak{L}_{Q}(j)$ are the $j$-th filtered probability spaces, the free blocks of the free filterization $\mathfrak{L}_{Q}(\mathbb{Z})$ of $Q$.

Furthermore, one obtains that

$$
\begin{aligned}
& \mathfrak{L}_{Q}(n, \mathbb{Z}) \stackrel{*-\text { iso }}{=} \underset{l=1}{\star}\left(\underset{j \in \mathbb{Z}}{\star}\left(L(\mathbb{Z})_{l} \otimes_{\mathbb{C}} \mathfrak{L}_{Q}(j)\right)\right)
\end{aligned}
$$

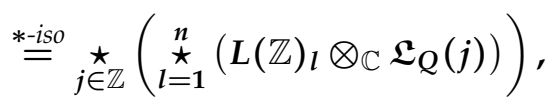

with

$$
L(\mathbb{Z})_{l}=L(\mathbb{Z}), \text { for all } l=1, \ldots, n,
$$

for all $n \in \mathbb{N}_{\infty}^{>1}$.

Proof. The structure theorem (10.7) are immediately obtained by (9.7) with help of (10.2) and (10.3), under the assumption (10.6). In addition, the structure theorem (10.8) is shown by (9.7) and (10.3)'.

The above isomorphism theorems (10.7) and (10.8) let us have the following corollary.

Corollary 8. Let $n \in \mathbb{N}_{\infty}^{>1}$, and let $\mathfrak{L}_{Q}(n, \mathbb{Z})$ be the $L\left(F_{n}\right)$-free filterization of $Q$.

If $n=n_{1}+\ldots+n_{k}$ as in (10.6) in $\mathbb{N}_{\infty}$, then

$$
\mathfrak{L}_{Q}(n, \mathbb{Z}) \stackrel{*-\text { iso }}{=} \underset{l=1}{\star \star}\left(\mathfrak{L}_{Q}\left(n_{l}, \mathbb{Z}\right)\right),
$$

where $\mathfrak{L}_{Q}\left(n_{l}, \mathbb{Z}\right)$ are the $L\left(F_{n_{l}}\right)$-free filterizations of $Q$, for all $l=1, \ldots, k$.

We have that 


$$
\mathfrak{L}_{Q}(n, \mathbb{Z}) \stackrel{* \text {-iso }}{=} \underset{l=1}{\star}\left(\mathfrak{L}_{Q}(\mathbf{1}, \mathbb{Z})\right),
$$

where $\mathfrak{L}_{Q}(\mathbf{1}, \mathbb{Z})$ is the $L(\mathbb{Z})$-free filterization $\mathfrak{L}_{Q}^{L(\mathbb{Z})}(\mathbb{Z})$ of $Q$.

Proof. By (10.7), one has that

$$
\begin{aligned}
& \mathfrak{L}_{Q}(n, \mathbb{Z})=\underset{l=1}{\star}\left(\underset{j \in \mathbb{Z}}{\star}\left(L\left(F_{n_{l}}\right) \otimes_{\mathbb{C}} \mathfrak{L}_{Q}(j)\right)\right) \\
& =\underset{l=1}{\star} \mathfrak{L}_{Q}\left(n_{l}, \mathbb{Z}\right),
\end{aligned}
$$

under (10.6). Therefore, the statement (10.9) holds.

In addition, the statement (10.10) holds as a special case of (10.9).

From below, let's fix $n \in \mathbb{N}_{\infty}^{>1}$ and the corresponding $L\left(F_{n}\right)$-free filterization $\mathfrak{L}_{Q}(n, \mathbb{Z})$.

Corollary 9. Let $\boldsymbol{T}_{l}=g_{l} \otimes \boldsymbol{U}_{j_{l}}^{n_{l}} \in \mathfrak{L}_{Q}(n, \mathbb{Z})$, where $g_{l} \in \boldsymbol{F}_{n}$ (and hence, they are generating operators of $L\left(F_{n}\right)$ ), and $\boldsymbol{U}_{j_{l}} \in \mathfrak{L}_{Q}\left(j_{l}\right)$ are semicircular elements in the free filterization $\mathfrak{L}_{Q}(\mathbb{Z})$, and $\boldsymbol{n}_{\boldsymbol{l}} \in \mathbb{N}$, for all $\boldsymbol{l}=\mathbf{1}$, $\ldots, N$, for $N \in \mathbb{N}$. Let

$$
T=\prod_{l=1}^{N} T_{l} \in \mathfrak{L}_{Q}(n, \mathbb{Z})
$$

Assume first that $N=\mathbf{1}$ in $\mathbb{N}$, and hence $T=g_{1} \otimes U_{j_{1}}^{n_{1}} \in \mathfrak{L}_{Q}(n, \mathbb{Z})$. Then,

$$
\tau^{L\left(F_{n}\right)}\left(T^{k}\right)=\tau^{L\left(F_{n}\right)}\left(\left(T^{*}\right)^{k}\right)= \begin{cases}\omega_{k n_{1}} c_{\frac{k n_{1}}{2}} & \text { if } g_{1}=e_{n} \\ 0 & \text { if } g_{1} \neq e_{n}\end{cases}
$$

for all $k \in \mathbb{N}$, where $\boldsymbol{e}_{n}$ is the group-identity of $\boldsymbol{F}_{n}$ (and, hence, the identity element of $L\left(F_{n}\right)$ ).

Suppose $N>\mathbf{1}$ in $\mathbb{N}$, and assume that $\boldsymbol{T}$ is in the sense of (10.11) in $\mathfrak{L}_{Q}(n, \mathbb{Z})$, and the corresponding $N$-tuple $\left(j_{1}, \ldots, j_{N}\right)$ is alternating in $\mathbb{Z}$. Then,

$$
\begin{aligned}
\tau^{L\left(F_{n}\right)}(T) & = \begin{cases}\prod_{l=1}^{N} \omega_{n_{l}} c_{\frac{n_{l}}{2}} & \text { if } g_{l}=e_{n}, \forall l=1, \ldots, N \\
0 & \text { otherwise, }\end{cases} \\
& =\tau^{L\left(F_{n}\right)}\left(T^{*}\right) .
\end{aligned}
$$

Under the same hypothesis of (10.13), assume further that $j_{1} \neq j_{N}$ in $\mathbb{Z}$. Then,

$$
\begin{aligned}
\tau^{L\left(F_{n}\right)}\left(T^{k}\right) & = \begin{cases}\prod_{l=1}^{N}\left(\omega_{n_{l}} c_{\frac{n_{l}}{2}}\right)^{k} & \begin{array}{l}
\text { if } g_{l}=e_{n}, \forall l=1, \ldots, N, \\
0
\end{array} \\
& \text { otherwise, }\end{cases} \\
& =\tau^{L\left(F_{n}\right)}\left(\left(T^{*}\right)^{k}\right),
\end{aligned}
$$

for all $k \in \mathbb{N}$.

Proof. Let $T=g_{1} \otimes U_{j_{1}}^{n_{1}} \in \mathfrak{L}_{Q}(n, \mathbb{Z})$, for $g_{1} \in F_{n}, U_{j_{1}} \in \mathfrak{L}_{Q}\left(j_{1}\right)$. Then, this operator $T$ is contained in the free block $\mathfrak{L}_{Q}^{L\left(F_{n}\right)}\left(j_{1}\right)$ in the $L\left(F_{n}\right)$-free filterization $\mathfrak{L}_{Q}(n, \mathbb{Z})$. Thus,

$$
T^{k}=\left(g_{1} \otimes U_{j_{1}}^{n_{1}}\right)^{k}=g_{1}^{k} \otimes U_{j_{1}}^{k n_{1}} \in \mathfrak{L}_{Q}^{L\left(F_{n}\right)}\left(j_{1}\right),
$$


in $\mathfrak{L}_{Q}(n, \mathbb{Z})$, and, hence,

$$
\left(T^{*}\right)^{k}=g_{1}^{-k} \otimes U_{j_{1}}^{k n_{1}} \in \mathfrak{L}_{Q}^{L\left(F_{n}\right)}\left(j_{1}\right),
$$

for all $k \in \mathbb{N}$, where $g_{1}^{-k}=\left(g_{1}^{-1}\right)^{k}$ in $F_{n}$ (and, hence, it is identical to $\left(g_{1}^{*}\right)^{k}$ in $L\left(F_{n}\right)$ ). Therefore, one can get that

by (8.17) and (8.18)

$$
\tau^{L\left(F_{n}\right)}\left(T^{k}\right)=\left(\omega_{k n_{1}} c_{\frac{k n_{1}}{2}}\right) \operatorname{tr}_{n}\left(g_{1}^{k}\right)
$$

where $\delta$ is the Kronecker delta

$$
=\left(\omega_{k n_{1}} c_{\frac{k n_{1}}{2}}\right) \delta_{g_{1}^{k}, e_{n}}
$$

for all $k \in \mathbb{N}$.

$$
= \begin{cases}\omega_{k n_{1}} c_{\frac{k n_{1}}{2}} & \text { if } g_{1}=e_{n} \text { in } F_{n}, \\ 0 & \text { if } g_{1} \neq e_{n} \text { in } F_{n}\end{cases}
$$

Similarly, one obtains that

for all $k \in \mathbb{N}$.

$$
\tau^{L\left(F_{n}\right)}\left(\left(T^{*}\right)^{k}\right)= \begin{cases}\omega_{k n_{1}} c_{\frac{k n_{1}}{2}} & \text { if } g_{1}^{-1}=e_{n} \text { in } F_{n} \\ 0 & \text { if } g_{1}^{-1} \neq e_{n} \text { in } F_{n},\end{cases}
$$

Note that the conditions for (10.15) and (10.16) are obtained by the very construction of free groups. For example, the generators of free groups have no relations.

Therefore, by (10.15) and (10.16), the statement (10.12) holds true.

Now, let $T$ be in the sense of (10.11), and suppose $\left(j_{1}, \ldots, j_{N}\right)$ is an alternating $N$-tuple in $\mathbb{Z}$. Then, this operator $T$ forms a free reduced word in $\mathfrak{L}_{Q}(n, \mathbb{Z})$. Thus, by (8.17) and (8.18), one can get that

$$
\begin{gathered}
\tau^{L\left(F_{n}\right)}(T)=\prod_{l=1}^{N} \tau_{j_{l}}^{L\left(F_{n}\right)}\left(g_{l} \otimes U_{j_{l}}^{n_{l}}\right) \\
=\prod_{l=1}^{N} t r_{n}\left(g_{l}\right)\left(\omega_{n_{l}} c \frac{n_{l}}{2}\right)=\prod_{l=1}^{N} \delta_{g_{l}, e_{n}} \omega_{n_{l}} c \frac{n_{l}}{2} .
\end{gathered}
$$

Similarly,

$$
\tau^{L\left(F_{n}\right)}\left(T^{*}\right)=\prod_{l=1}^{N} \delta_{g_{l}^{-1}, e_{n}} \omega_{n_{l}} c_{\frac{n_{l}}{2}} .
$$

Therefore, by (10.17) and (10.18), the statement (10.13) also holds true.

Finally, a given operator $T$ of (10.11) is a free reduced word of the $L\left(F_{n}\right)$-free filterization $\mathfrak{L}_{Q}(n, \mathbb{Z})$, as in (10.13). Assume more now that

$$
j_{1} \neq j_{N} \text { in } \mathbb{Z} \text {. }
$$

Then, one can check that the operators $T^{k}$ and $\left(T^{*}\right)^{k}$ are free reduced words in $\mathfrak{L}_{Q}(n, \mathbb{Z})$. It allows us to have

$$
\tau^{L\left(F_{n}\right)}\left(T^{k}\right)=\left(\tau^{L\left(F_{n}\right)}(T)\right)^{k}
$$

and

$$
\tau^{L\left(F_{n}\right)}\left(\left(T^{*}\right)^{k}\right)=\left(\tau^{L\left(F_{n}\right)}\left(T^{*}\right)\right)^{k}
$$

for all $k \in \mathbb{N}$.

Therefore, by (10.13), the statement (10.14) holds true. 
The above corollary characterizes how to compute free-distributional data. Different from the above corollary, we also obtain the following free-moment computations from (9.14) and (9.15).

Corollary 10. Let $j \in \mathbb{Z}$, and $N \in \mathbb{N}$ be fixed, and let $\mathfrak{L}_{Q}(n, \mathbb{Z})$ be the given $L\left(F_{n}\right)$-free filterization of $Q$. Let

$$
y_{l}=g_{l} \otimes U_{j}^{n_{l}} \in \mathfrak{L}_{Q}(n, \mathbb{Z}), \text { with } n_{l} \in \mathbb{N},
$$

where $g_{l} \in L\left(F_{n}\right)$, for all $l=1, \ldots, N$, and let

$$
T=\prod_{l=1}^{N} y_{l} \in \mathfrak{L}_{Q}(n, \mathbb{Z})
$$

Then,

$$
\tau^{L\left(F_{n}\right)}(T)=\tau^{L\left(F_{n}\right)}\left(T^{*}\right)=\delta_{g_{T}, e_{n}} \omega_{n_{T} \frac{c_{\frac{n}{2}}}{2}}
$$

with

$$
g_{T}=\prod_{l=1}^{N} g_{l} \in F_{n} \text {, and } n_{T}=\sum_{l=1}^{N} n_{l} \text { in } \mathbb{N} \text {. }
$$

Moreover, we have that:

$$
\text { if } N=1, \text { then } \tau^{L\left(F_{n}\right)}(T)=\delta_{g_{1}, e_{n}} \omega_{n_{1}} c_{\frac{n_{1}}{2}} .
$$

if $N>\mathbf{1}$ is odd in $\mathbb{N}$, and if

$$
\left(g_{1}, \ldots, g_{N}\right) \in F_{n} \times \cdots \times F_{n}
$$

contains either no identity element $e_{n}$ as its entry, or even-many identity elements as its entries, then $\tau^{L}\left(F_{n}\right)(T)=0$. if $N$ is even in $\mathbb{N}$, and if there exists $x_{1}, \ldots, x_{\frac{N}{2}} \in F_{n}$, such that

$$
\left(g_{1}, g_{2}, \ldots, g_{N}\right)=\left(x_{1}, x_{1}^{-1}, x_{2}, x_{2}^{-1}, \ldots, x_{\frac{N}{2}}, x_{\frac{N}{2}}^{-1}\right)
$$

in $F_{n} \times \cdots \times F_{n}$, then

$$
\tau^{L\left(F_{n}\right)}(T)=\omega_{n_{T}} c_{\frac{n_{T}}{2}}=\tau^{L\left(F_{n}\right)}\left(T^{*}\right),
$$

where $\boldsymbol{n}_{T}$ is in the sense of (10.20). For the fixed even number $\mathbf{N}$,

$$
\tau^{L\left(F_{n}\right)}(T)=0=\tau^{L\left(F_{n}\right)}\left(T^{*}\right),
$$

otherwise.

Proof. Suppose $T$ is an operator (10.19) in $\mathfrak{L}_{Q}(n, \mathbb{Z})$. From the very construction (10.19) of $T$, one can realize that this operator $T$ is contained in the free block $\mathfrak{L}_{Q}^{L\left(F_{n}\right)}(j)$ of $\mathfrak{L}_{Q}(n, \mathbb{Z})$, for the fixed integer $j$, since

$$
T=\left(\prod_{l=1}^{N} g_{l}\right) \otimes U_{j}^{n_{T}}, \text { with } n_{T}=\sum_{l=1}^{N} n_{l} \in \mathbb{N},
$$

is contained in $\mathfrak{L}_{Q}^{L\left(F_{n}\right)}(j)$.

Therefore by (9.14) and (9.15), one has that

$$
\tau^{L\left(F_{n}\right)}(T)=\omega_{n_{T}} c_{\frac{n_{T}}{2}} \operatorname{tr}_{n}\left(\prod_{l=1}^{N} g_{l}\right) .
$$


Similarly,

$$
\tau^{L\left(F_{n}\right)}\left(T^{*}\right)=\omega_{n_{T}} c_{\frac{n_{T}}{2}} \operatorname{tr}_{n}\left(\left(\prod_{l=1}^{N} g_{l}\right)^{-1}\right) .
$$

Therefore, the free-distributional data (10.20) holds.

The statements (10.21), (10.22) and (10.23) are nothing but a re-expression of (10.20).

The above free-distributional data (10.12), (10.13), (10.14) and (10.20) provide the general ways to compute free distributions of operators in the $L\left(F_{n}\right)$-free filterization $\mathfrak{L}_{Q}(n, \mathbb{Z})$, for $n \in \mathbb{N}_{\infty}^{>1}$.

Conflicts of Interest: The author declares no conflict of interest.

\section{References}

1. Radulescu, F. Random Matrices, Amalgamated Free Products and Subfactors of the $C^{*}$-Algebra of a Free Group of Nonsingular Index. Invent. Math. 1994, 115, 347-389.

2. Free Probability Theory. In The Fields Institute Communication; Voiculescu, D., Ed.; Amarican Mathmatical Society: Providence, RI, USA, 1997; Volume 12, ISBN 0-8218-0675-0.

3. Voiculescu, D.; Dykemma, K.; Nica, A. Free Random Variables; CRM Monograph Series; Amarican Mathmatical Society: Providence, RI, USA, 1992, Volume 1.

4. Cho, I. Semicircular-Like Laws and the Semicircular Law Induced by Orthogonal Projections. Complex Anal. Oper. Theory 2015, submitted.

5. Cho, I. $p$-Adic Free Stochastic Integrals for $p$-Adic Weighted-Semicircular Motions Determined by Primes $p$. Lib. Math. 2016, 36, 65-110.

6. Cho, I. Free Semicircular Families in Free Product Banach $*$-Algebras Induced by $p$-Adic Number Fields over Primes p. Complex Anal. Oper. Theory 2017, 11, 507-565.

7. Cho, I.; Gillespie, T. Free Probability on the Hecke Algebra. Complex Anal. Oper. Theory 2014, doi:10.1007/s11785-014-0403-1.

8. Cho, I. Representations and Corresponding Operators Induced by Hecke Algebras. Complex Anal. Oper. Theory 2016, 10, 437-477.

9. Speicher, R. Combinatorial Theory of the Free Product with Amalgamation and Operator-Valued Free Probability Theory. Mem. Am. Math. Soc. 1998, 132, doi:10.1090/memo/0627.

10. Gilles, P. Introduction to Operator Space Theory; Cambridge University Press: Cambridge, UK, 2003, ISBN 978-0-521-81165-1.

11. Blackadar, B. Operator Algebras; Springer: Berlin, Germany, 2013; ISBN 3-540-28486-9.

(C) 2017 by the author. Licensee MDPI, Basel, Switzerland. This article is an open access article distributed under the terms and conditions of the Creative Commons Attribution (CC BY) license (http:/ / creativecommons.org/licenses/by/4.0/). 